\title{
LAGRANGIAN CONFIGURATIONS AND SYMPLECTIC CROSS-RATIOS
}

\author{
CHARLES H. CONLEY AND VALENTIN OVSIENKO
}

\begin{abstract}
We consider moduli spaces of cyclic configurations of $N$ lines in a $2 n$-dimensional symplectic vector space, such that every set of $n$ consecutive lines generates a Lagrangian subspace. We study geometric and combinatorial problems related to these moduli spaces, and prove that they are isomorphic to quotients of spaces of symmetric linear difference operators with monodromy -1 .

The symplectic cross-ratio is an invariant of two pairs of 1-dimensional subspaces of a symplectic vector space. For $N=2 n+2$, the moduli space of Lagrangian configurations is parametrized by $n+1$ symplectic cross-ratios. These cross-ratios satisfy a single remarkable relation, related to tridiagonal determinants and continuants, given by the Pfaffian of a Gram matrix.
\end{abstract}

\section{CONTENTS}

1. Introduction

1.1. Example: hexagons in $\mathbb{K}^{3}$

1.2. Example: the Gauss relations

1.3. Outline of results

2. Lagrangian configurations and their moduli spaces

2.1. Symplectic cross-ratios

2.2. Gram matrices

2.3. Continuous invariants

2.4. Opposite configurations and sign invariants

2.5. Dimensions and standard configurations

2.6. The case $N>2 n+2$

3. The main results

3.1. The cases $N=2 n$ and $N=2 n+1$

3.2. The first non-trivial case: $N=2 n+2$

3.3. The cyclic continuant

4. Pfaffians and the proof of Theorem 1

4.1. Tridiagonal determinants and the proof of Theorem 1(i)

4.2. Proof of Theorem 1(ii)

4.3. Proof of Theorem 1(iii)

5. Normalized configurations

5.1. The case of $n$ even and $\mathbb{K}=\mathbb{C}$

5.2. The case of $n$ even and $\mathbb{K}=\mathbb{R}$

5.3. The case of $n$ odd and $\mathbb{K}=\mathbb{C}$

5.4. The case of $n$ odd and $\mathbb{K}=\mathbb{R}$

2

6. Symmetric linear difference equations and the closure of $\mathcal{L}_{n, N}(\mathbb{K}) \quad 20$

6.1. Linear difference operators $\quad 20$

6.2. Linear difference equations $\quad 21$

6.3. Periodic operators, monodromy, and Lagrangian configurations $\quad 23$

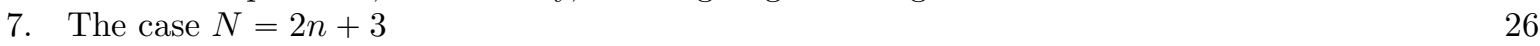

$\begin{array}{ll}\text { References } & 28\end{array}$ 


\section{INTRODUCTION}

Throughout this article, $\mathbb{K}$ will denote either $\mathbb{R}$ or $\mathbb{C},\left\{e_{1}, \ldots, e_{n}, f_{1}, \ldots, f_{n}\right\}$ will be the standard basis of $\mathbb{K}^{2 n}$, and $\omega$ will be the standard symplectic form on $\mathbb{K}^{2 n}$ : for $1 \leqslant i, j \leqslant n$,

$$
\omega\left(e_{i}, e_{j}\right)=0, \quad \omega\left(e_{i}, f_{j}\right)=\delta_{i j}, \quad \omega\left(f_{i}, f_{j}\right)=0 .
$$

We define the symplectic group $\operatorname{Sp}(2 n, \mathbb{K})$ with respect to $\omega$. Our topic of study is configurations of 1 -dimensional subspaces of $\mathbb{K}^{2 n}$ modulo the action of $\operatorname{Sp}(2 n, \mathbb{K})$.

Definition. For $N \geqslant 2 n$, define an $(n, N)$-Lagrangian configuration over $\mathbb{K}$ to be a cyclically ordered $N$ tuple $\left(X_{1}, \ldots, X_{N}\right)$ of lines through the origin in the symplectic space $\mathbb{K}^{2 n}$ with the following properties (by cyclically ordered, we mean that the indices are read modulo $N$ ):

(i) Every $n$ consecutive lines span a Lagrangian subspace: $\left\langle X_{i+1}, \ldots, X_{i+n}\right\rangle$ is Lagrangian for all $i$.

(ii) Every $2 n$ consecutive lines span the entire symplectic space: $\left\langle X_{i+1}, \ldots, X_{i+2 n}\right\rangle=\mathbb{K}^{2 n}$ for all $i$.

Lagrangian configurations in the same orbit of $\operatorname{Sp}(2 n, \mathbb{K})$ are said to be equivalent.

We have formulated our results in the symplectic setting, but we may just as well speak of Legendrian configurations of points in the contact projective space $\mathbb{K}^{2 n-1}$. Let us mention that Legendrian configurations in $\mathbb{R P}^{3}$ may be viewed as discrete analogs of Legendrian knots. In another direction, one may consider cyclically ordered $N$-tuples of Lagrangian subspaces $\left(L_{1}, L_{2}, \ldots, L_{N}\right)$ in $\mathbb{R}^{2 n}$ such that every two consecutive subspaces $L_{i}$ and $L_{i+1}$ are "maximally non-transversal". These configurations are in some sense dual to Lagrangian configurations, and the Maslov index may be applied to study them; see 2 . Continuous versions were treated in [16].

Suppose that an arbitrary cyclically ordered $N$-tuple $\left(X_{1}, \ldots, X_{N}\right)$ of lines through the origin in $\mathbb{K}^{2 n}$ has Property (i) above. We will see in Lemma 2.2 that then it has Property (ii) if and only if the subspace $\left\langle X_{i}, X_{i+n}\right\rangle$ is not isotropic for any $i$. It turns out that $\operatorname{Sp}(2 n, \mathbb{K})$ does not act freely on all Lagrangian configurations, but for $N>2 n$, it does act freely on configurations in which $\left\langle X_{i}, X_{j}\right\rangle$ is not isotropic except when forced to be so by Property (i); see Proposition 2.7. We refer to such configurations as generic:

Definition. An $(n, N)$-Lagrangian configuration $\left(X_{1}, \ldots, X_{N}\right)$ is generic if $\left\langle X_{i}, X_{j}\right\rangle$ is non-isotropic whenever the $N$-cyclic distance between $i$ and $j$ is at least $n$, that is, $i-j$ is not congruent to any of $0, \pm 1, \pm 2, \ldots, \pm(n-1)$ modulo $N$. We denote the $\operatorname{Sp}(2 n, \mathbb{K})$-moduli space of generic $(n, N)$-configurations over $\mathbb{K}$ by $\mathcal{L}_{n, N}(\mathbb{K})$.

The space $\mathcal{L}_{n, N}(\mathbb{K})$ is the main object of our study. We will see that $\operatorname{Sp}(2 n, \mathbb{K})$ acts freely on generic configurations, implying that $\mathcal{L}_{n, N}(\mathbb{K})$ is a variety of dimension $n(N-2 n-1)$, and moreover, $\mathcal{L}_{n, N}(\mathbb{R})$ and $\mathcal{L}_{n, N}(\mathbb{C})$ are smooth real and complex manifolds of this dimension, respectively. We construct a collection of symplectic cross-ratios which are $\operatorname{Sp}(2 n, \mathbb{K})$-invariants of Lagrangian configurations over $\mathbb{K}$, and in some cases show that these cross-ratios form a coordinate ring on $\mathcal{L}_{n, N}(\mathbb{K})$. They satisfy certain relations, which we calculate explicitly as Pfaffians for $N=2 n+2$, the simplest non-trivial case. These Pfaffians are closely related to the classical determinants of continued fractions known as continuants; see 3 .

Observe that for $n=1$ the Lagrangian condition is vacuous, so $\mathcal{L}_{1, N}(\mathbb{K})$ is essentially the classical moduli space $\mathcal{M}_{0, N}$ of configurations of $N$ points on the projective line. We regard $\mathcal{L}_{n, N}(\mathbb{K})$ as a multidimensional symplectic variant of $\mathcal{M}_{0, N}$. The only previously studied configurations in symplectic space we know of are triangles and skew lines; see [22] and Section 2.8 of [15]. To the best of our knowledge, Lagrangian configurations have not been considered before. We believe that they deserve further study; in particular, it would be interesting to investigate the topology of $\mathcal{L}_{n, N}(\mathbb{K})$. It seems plausible that the topological invariants of Legendrian knots, for instance, the Maslov class and the Bennequin invariant, as well as more general invariants, can be expressed in terms of cross-ratios of a Lagrangian configuration. 
Relations to dynamical systems also seem promising. Moduli spaces of cyclic configurations of points in $\mathbb{R P}^{n}$ (without any Legendrian condition) carry a family of discrete integrable systems, including the pentagram map and its generalizations; see [6, 8, 9, 18, 19, 21. We believe that $\mathcal{L}_{n, N}(\mathbb{K})$ also supports interesting discrete dynamical systems.

1.1. Example: hexagons in $\mathbb{K} \mathbb{P}^{3}$. Our main geometric result is a description of $\mathcal{L}_{n, 2 n+2}$. The simplest case, $\mathcal{L}_{1,4}(\mathbb{K})$, is the moduli space of quadrilaterals in $\mathbb{K} \mathbb{P}^{1}$. It has been known since ancient times to be 1-dimensional and parametrized by the classical cross-ratio. Therefore the first new case is $\mathcal{L}_{2,6}(\mathbb{K})$, the $\operatorname{Sp}(4, \mathbb{K})$-moduli space of generic $(2,6)$-Lagrangian configurations, i.e., Legendrian hexagons in $\mathbb{K} \mathbb{P}^{3}$.

Given any $(2,6)$-configuration, choosing a non-zero point on each of the six lines gives a hexagon $\left(x_{0}, \ldots, x_{5}\right)$ in $\mathbb{K}^{4}$. It turns out to be natural to regard this hexagon as the 6 -antiperiodic sequence $\left(x_{i}\right)_{i \in \mathbb{Z}}$ defined by $x_{i \pm 6}:=-x_{i}$. Then

$$
\omega\left(x_{i}, x_{i+3}\right)=\omega\left(x_{i+3}, x_{i+6}\right), \quad \omega\left(x_{i}, x_{i+2}\right)=\omega\left(x_{i+2}, x_{i+6}\right) .
$$

The sequences $\left(\omega\left(x_{i}, x_{i+2}\right)\right)_{i \in \mathbb{Z}}$ and $\left(\omega\left(x_{i}, x_{i+3}\right)\right)_{i \in \mathbb{Z}}$ are 6-periodic and 3-periodic, respectively.

The Lagrangian conditions are $\omega\left(x_{i}, x_{i+1}\right)=0$ and $\omega\left(x_{i}, x_{i+2}\right) \neq 0$. Thus we may say that we are considering hexagons whose sides are of "symplectic length zero", but whose "symplectic subdiameters" are non-zero. The generic configurations are those with non-zero "symplectic diameters": $\omega\left(x_{i}, x_{i+3}\right) \neq 0$.

The three "diametric symplectic cross-ratios"

$$
c_{i}:=\frac{\omega\left(x_{i}, x_{i+3}\right) \omega\left(x_{i+1}, x_{i+4}\right)}{\omega\left(x_{i}, x_{i+4}\right) \omega\left(x_{i+1}, x_{i+3}\right)}, \quad i=0,1,2,
$$

depend only on the original configuration of lines, not the choice of points $x_{i}$, and are symplectic invariants. We will see that they form an essentially complete set of invariants parametrizing $\mathcal{L}_{2,6}(\mathbb{K})$. As an example, we illustrate $c_{0}$ by the following diagram.

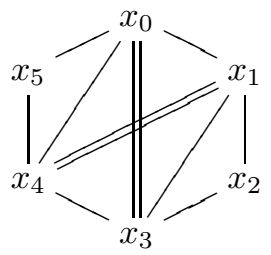

Figure 1. The cross-ratio $c_{0}=\frac{\omega\left(x_{0}, x_{3}\right) \omega\left(x_{1}, x_{4}\right)}{\omega\left(x_{0}, x_{4}\right) \omega\left(x_{1}, x_{3}\right)}$ on $\mathcal{L}_{2,6}(\mathbb{K})$.

Observe that the space of all $(2,6)$-Lagrangian configurations is 12-dimensional: there are three degrees of freedom for each of the six points, and six Lagrangian conditions. As mentioned earlier, the 10-dimensional group $\operatorname{Sp}(4, \mathbb{K})$ acts freely on the generic configurations, so $\mathcal{L}_{2,6}(\mathbb{K})$ is 2-dimensional. Therefore the three cross-ratios cannot be independent. In fact, they satisfy the relation

$$
\frac{1}{c_{0}}+\frac{1}{c_{1}}+\frac{1}{c_{2}}=1
$$

Theorem 1 resolves the situation, describing $\mathcal{L}_{2,6}(\mathbb{K})$ completely:

- (1.1) is the only relation on the cross-ratios: any three non-zero $\mathbb{K}$-scalars $c_{0}, c_{1}, c_{2}$ satisfying it are the cross-ratios of a generic Lagrangian configuration.

- The cross-ratios are complete continuous invariants for Lagrangian configurations: equivalent configurations have the same cross-ratios, and any two configurations with the same cross-ratios are equivalent if $\mathbb{K}=\mathbb{C}$, and either equivalent or opposite (see Section 2.4) if $\mathbb{K}=\mathbb{R}$.

These results may be reformulated in terms of normalized configurations as follows. For $\mathbb{K}=\mathbb{C}$, the $x_{i}$ can be rescaled so that the symplectic subdiameters $\omega\left(x_{i}, x_{i+2}\right)$ are all 1 . Then the symplectic diameters 
$a_{i}:=\omega\left(x_{i}, x_{i+3}\right)$ become, up to an overall choice of sign, symplectic invariants. Indeed, here $c_{i}=a_{i} a_{i+1}$, so (1.1) becomes

$$
a_{0} a_{1} a_{2}=a_{0}+a_{1}+a_{2} .
$$

For $\mathbb{K}=\mathbb{R}$, it may happen that only complex rescalings can bring all subdiameters to 1 . However, the required scale factors are always either real or pure imaginary. There are four possibilities: the normalized $x_{i}$ with $i$ even are either all real or all pure imaginary, and similarly for $i$ odd. If the normalized $x_{i}$ are all real or all imaginary, then the $a_{i}$ are all real, while if the normalized $x_{i}$ are half real and half imaginary, then the $a_{i}$ are all imaginary.

Let us remark that up to permutation, $\left(c_{0}, c_{1}, c_{2}\right)=(2,3,6)$ is the only Egyptian fraction solution of (1.1). It arises from $\left(a_{0}, a_{1}, a_{2}\right)=(1,2,3)$, the only positive integer solution of (1.2). Integer solutions of the multi-dimensional analogs of these relations are discussed in [3, 17.

1.2. Example: the Gauss relations. In the final section of this article we make some initial remarks on the relations between the symplectic cross-ratios of $\mathcal{L}_{n, 2 n+3}$. Historically, the earliest examples of relations between cross-ratios arose in Gauss' pentagramma mirificum [7], which is $\mathcal{M}_{0,5}(\mathbb{K})$, or equivalently, $\mathcal{L}_{1,5}(\mathbb{K})$, the moduli space of pentagons in $\mathbb{K} \mathbb{P}^{1}$.

As we did for Legendrian hexagons, given five points in $\mathbb{K P}^{1}$, lift them to non-zero points $x_{0}, \cdots, x_{4}$ in $\mathbb{K}^{2}$ and extend to a 5 -antiperiodic sequence $\left(x_{i}\right)_{i \in \mathbb{Z}}$ via $x_{i \pm 5}:=-x_{i}$. Gauss discovered that the 5 -periodic sequence of cross-ratios $d_{i}:=\omega\left(x_{i-3}, x_{i}\right) \omega\left(x_{i-2}, x_{i-1}\right) / \omega\left(x_{i-3}, x_{i-2}\right) \omega\left(x_{i-1}, x_{i}\right)$ satisfy the relations

$$
d_{i} d_{i+1}=d_{i+3}+1 .
$$

These five Gauss relations completely determine the varietal structure of $\mathcal{M}_{0,5}$. They can be rewritten in the remarkable form

$$
\left(\begin{array}{ll}
d_{0} & 1 \\
-1 & 0
\end{array}\right)\left(\begin{array}{cc}
d_{1} & 1 \\
-1 & 0
\end{array}\right)\left(\begin{array}{cc}
d_{2} & 1 \\
-1 & 0
\end{array}\right)\left(\begin{array}{cc}
d_{3} & 1 \\
-1 & 0
\end{array}\right)\left(\begin{array}{cc}
d_{4} & 1 \\
-1 & 0
\end{array}\right)=\left(\begin{array}{cc}
-1 & 0 \\
0 & -1
\end{array}\right) .
$$

This relates the topic to two classical subjects: the theory of continued fractions and the theory of linear difference equations. The Gauss relations were the main motivation for Coxeter 4 to develop the notion of frieze patterns, relating projective geometry to combinatorics. Friezes provide a special parametrization of $\mathcal{M}_{0, N}$; see [13] and the appendix of [14].

We regard the relations between the symplectic cross-ratios of $\mathcal{L}_{n, N}$ as multi-dimensional analogs of the Gauss relations. Building on preliminary versions of this article, Morier-Genoud [12] has studied the combinatorial aspects of $\mathcal{L}_{2, N}(\mathbb{C})$, the moduli space of Legendrian $N$-gons in $\mathbb{C P}^{3}$. Her work indicates that in general, $\mathcal{L}_{n, N}(\mathbb{K})$ has a rich combinatorial structure related to friezes.

1.3. Outline of results. It is natural to ask for a coordinate system on the moduli space $\mathcal{L}_{n, N}(\mathbb{K})$ of $(n, N)$-Lagrangian configurations. In this article we show that this question is vacuous when $N$ is $2 n$ or $2 n+1$, and answer it when $N$ is $2 n+2$. Our coordinates are given by the symplectic cross-ratio, a direct analog of the classical cross-ratio: we show that $\mathcal{L}_{n, 2 n+2}(\mathbb{K})$ is parametrized by symplectic cross-ratios and determine its structure as an algebraic variety. We expect that symplectic cross-ratios parametrize $\mathcal{L}_{n, N}(\mathbb{K})$ for all $N$. The exposition is organized as follows.

In Section 2 we define symplectic cross-ratios and show that they provide continuous invariants on $(n, N)$-Lagrangian configurations for $N \geqslant 2 n+2$. We also deduce the dimension of $\mathcal{L}_{n, N}(\mathbb{K})$ and define opposite configurations and equivalence classes, which over $\mathbb{R}$ are distinguished by sign invariants.

Section 3 contains our main geometric results, which we summarize here:

- $(n, 2 n)$-configurations are all generic and equivalent over both $\mathbb{C}$ and $\mathbb{R}$.

- $(n, 2 n+1)$-configurations are all generic. Over $\mathbb{C}$ they are all equivalent, and over $\mathbb{R}$ there are two equivalence classes, which are opposite. 
- $(n, 2 n+2)$-configurations admit $n+1$ diametric symplectic cross-ratios $c_{0}, \ldots, c_{n}$ :

$$
c_{i}:=\frac{\omega\left(x_{i}, x_{i+n+1}\right) \omega\left(x_{i+1}, x_{i+n+2}\right)}{\omega\left(x_{i}, x_{i+n+2}\right) \omega\left(x_{i+1}, x_{i+n+1}\right)},
$$

the $x_{i}$ being arbitrary non-zero points on the lines of the configuration.

Over $\mathbb{C}$, generic $(n, 2 n+2)$-configurations are equivalent if and only if they have the same diametric cross-ratios. Over $\mathbb{R}$, generic $(n, 2 n+2)$-configurations with the same cross-ratios are either equivalent or in opposite equivalence classes.

The moduli space $\mathcal{L}_{n, 2 n+2}(\mathbb{K})$ is $n$-dimensional. The $n+1$ cross-ratios satisfy the relation (3.2), and any collection of non-zero $\mathbb{K}$-scalars $\left(c_{0}, \ldots, c_{n}\right)$ satisfying (3.2) is the set of cross-ratios of an $(n, 2 n+2)$-configuration over $\mathbb{K}$. Thus the cross-ratios are coordinates describing $\mathcal{L}_{n, 2 n+2}(\mathbb{K})$ as an algebraic hypersurface in $\mathbb{K}^{n+1}$. These results are presented in Theorem 1 .

For $(n, 2 n+2)$-configurations the proof has several components and is given in Section 4 ,

In Section 5 we present certain normalized choices of the $x_{i}$ generalizing Section 1.1. For $n$ even there is an essentially unique choice such that the symplectic subdiameters $\omega\left(x_{i}, x_{i+n}\right)$ are all 1 . This normalization provides an alternate coordinate system on $\mathcal{L}_{n, 2 n+2}(\mathbb{K})$ : the symplectic diameters $a_{0}, \ldots, a_{n}$, where $a_{i}:=\omega\left(x_{i}, x_{i+n+1}\right)$. These diameters are determined up to an overall choice of sign, and for $\mathbb{K}=\mathbb{R}$ they are either all real or all pure imaginary. In this coordinate system the relation (3.2) can be written in terms of the celebrated classical determinants called continuants. This connection is emphasized in Section 3.3, see Theorem 2.

For $n$ odd, one cannot in general choose the points $x_{i}$ so that the symplectic subdiameters are all 1 , but one can choose them so that the subdiameters alternate between a scalar $\mu$ and its reciprocal, and the two alternating products of diameters are equal: $a_{0} a_{2} \cdots a_{n-1}=a_{1} a_{3} \cdots a_{n}$. Here $\mu$ is determined up to a $(2 n+2)^{\text {nd }}$ root of unity, and the $a_{i}$ are determined up to an overall $(n+1)^{\text {st }}$ root of unity.

An old idea of projective differential geometry consists in representing geometric objects such as curves or configurations of points via differential or difference operators. Following this approach, in Section [6] we realize the moduli space of Lagrangian configurations as the quotient by rescaling of the space of symmetric linear difference equations with periodic coefficients and antiperiodic solutions; see Theorem 3 ,

We conclude in Section 7 with a preliminary discussion of $\mathcal{L}_{n, 2 n+3}$, including a general result on normalizations in the case that $N / \operatorname{GCD}(n, N)$ is odd, and relations on cross-ratios for $\mathcal{L}_{2,7}$ and $\mathcal{L}_{3,9}$, the moduli spaces of generic Legendrian heptagons in $\mathbb{K} \mathbb{P}^{3}$ and Legendrian nonagons in $\mathbb{K} \mathbb{P}^{5}$.

\section{LAGRANGIAN CONFIGURATIONS AND THEIR MODULI SPACES}

In this section we collect some basic properties of Lagrangian configurations and the action of $\operatorname{Sp}(2 n, \mathbb{K})$ on them. We prove that the action is free on generic configurations and introduce two types of invariants: continuous invariants known as symplectic cross-ratios, and certain discrete sign invariants.

2.1. Symplectic cross-ratios. Consider two pairs of points in $\left(\mathbb{K}^{2 n}, \omega\right),\left(x_{1}, x_{2}\right)$ and $\left(y_{1}, y_{2}\right)$, such that $\omega\left(x_{1}, y_{2}\right)$ and $\omega\left(x_{2}, y_{1}\right)$ are non-zero. We define their symplectic cross-ratio to be

$$
\left[x_{1}, x_{2} ; y_{1}, y_{2}\right]:=\frac{\omega\left(x_{1}, y_{1}\right) \omega\left(x_{2}, y_{2}\right)}{\omega\left(x_{1}, y_{2}\right) \omega\left(x_{2}, y_{1}\right)} .
$$

The symplectic cross-ratio is obviously invariant with respect to both the action of the symplectic group $\operatorname{Sp}(2 n, \mathbb{K})$ and rescalings $x_{i} \mapsto \lambda_{i} x_{i}$ and $y_{i} \mapsto \mu_{i} y_{i}$. Therefore it is in fact a symplectic invariant of two pairs of 1 -dimensional subspaces in $\mathbb{K}^{2 n}$, or equivalently, of two pairs of points in $\mathbb{K} \mathbb{P}^{2 n-1}$. Observe the symmetries

$$
\left[x_{2}, x_{1} ; y_{1}, y_{2}\right]=\left[x_{1}, x_{2} ; y_{1}, y_{2}\right]^{-1}, \quad\left[y_{1}, y_{2} ; x_{1}, x_{2}\right]=\left[x_{1}, x_{2} ; y_{1}, y_{2}\right] .
$$

Remark. For $n>1$, (2.1) is not the only symplectic invariant of a quadruple ( $\left.\mathbb{K} x_{1}, \mathbb{K} x_{2}, \mathbb{K} y_{1}, \mathbb{K} y_{2}\right)$ of lines in $\mathbb{K}^{2 n}$. However, it is the only such invariant if 
- $\left\langle x_{1}, x_{2}\right\rangle$ and $\left\langle y_{1}, y_{2}\right\rangle$ are isotropic, i.e., $\omega\left(x_{1}, x_{2}\right)$ and $\omega\left(y_{1}, y_{2}\right)$ are 0 , and

- $\left(x_{1}, x_{2}, y_{1}, y_{2}\right)$ is generic under this condition.

In the 1-dimensional case, (2.1) is nothing but the classical cross-ratio of 4 points on the projective line. In affine coordinates, it is given by the usual formula:

$$
\left[x_{1}, x_{2} ; y_{1}, y_{2}\right]=\frac{\left(x_{1}-y_{1}\right)\left(x_{2}-y_{2}\right)}{\left(x_{1}-y_{2}\right)\left(x_{2}-y_{1}\right)}
$$

It is the unique $\operatorname{PSL}(2, \mathbb{K})$-invariant of $\left(x_{1}, x_{2}, y_{1}, y_{2}\right)$. Different partitions of the points into two pairs give six different cross-ratios, but any one of them determines the others.

Remark. The cross-ratio plays a fundamental role in many areas, from projective geometry to mathematical physics; for an overview, see [11. It is the discrete version of the Schwarzian derivative; see, e.g., 20]. Different versions of multi-dimensional symplectic cross-ratios have been considered. One is an invariant of a quadruples of Lagrangian planes related to the Maslov index; again, see 20 for a survey. Another is a unitary group invariant defined in the complex setting; see [5. The symplectic cross-ratio (2.1) is the most straightforward generalization of the 1-dimensional cross-ratio. It has been used to construct symplectic projective invariants in other settings; see, e.g., [15] (p. 367) and 22].

2.2. Gram matrices. Given a collection $x_{1}, \ldots, x_{m}$ of vectors in $\mathbb{K}^{2 n}$, we define their $\omega$-Gram matrix $\Omega\left(x_{1}, \ldots, x_{m}\right)$ to be the $m \times m$ matrix whose entries are their symplectic products:

$$
\Omega\left(x_{1}, \ldots, x_{m}\right)_{i j}:=\omega\left(x_{i}, x_{j}\right) .
$$

We will use the following standard lemma throughout the paper. Its proof is elementary and is omitted.

Lemma 2.1. Suppose that $x_{1}, \ldots, x_{m}$ and $x_{1}^{\prime}, \ldots, x_{m}^{\prime}$ are two collections of $m$ vectors in $\mathbb{K}^{2 n}$.

(i) $\Omega\left(x_{1}, \ldots, x_{m}\right)$ is of rank at most $2 n$.

(ii) $\Omega\left(x_{1}, \ldots, x_{m}\right)$ is of rank $2 n$ if and only if $\left\langle x_{1}, \ldots, x_{m}\right\rangle=\mathbb{K}^{2 n}$.

(iii) If $\Omega\left(x_{1}, \ldots, x_{m}\right)$ and $\Omega\left(x_{1}^{\prime}, \ldots, x_{m}^{\prime}\right)$ are equal and of rank $2 n$, then there is a unique symplectic transformation $T$ such that $T\left(x_{i}\right)=x_{i}^{\prime}$ for all $i$.

Lemma 2.2. Fix $N \geqslant 2 n$ and let $\left(x_{1}, \ldots, x_{N}\right)$ be an $N$-tuple of points in $\mathbb{K}^{2 n}$. Define $\left(x_{i}\right)_{i \in \mathbb{Z}}$ via $x_{i \pm N}:=-x_{i}$, and assume that $\left\langle x_{i+1}, \ldots, x_{i+n}\right\rangle$ is Lagrangian for all $i$. Then $\left(\mathbb{K} x_{1}, \ldots, \mathbb{K} x_{N}\right)$ is an $(n, N)$-Lagrangian configuration if and only if $\omega\left(x_{i}, x_{i+n}\right) \neq 0$ for all $i$.

Proof. We must show that $\left\langle x_{i+1}, \ldots, x_{i+2 n}\right\rangle=\mathbb{K}^{2 n}$ for all $i$ if and only if $\omega\left(x_{i}, x_{i+n}\right) \neq 0$ for all $i$. Consider the $\omega$-Gram matrix $\Omega^{i}:=\Omega\left(x_{i+1}, \ldots, x_{i+2 n}\right)$. By Lemma 2.1(ii), $\operatorname{det}\left(\Omega^{i}\right) \neq 0$ if and only if $x_{i+1}, \ldots, x_{i+2 n}$ form a basis of $\mathbb{K}^{2 n}$. The Lagrangian condition implies that the block $2 \times 2$ form of $\Omega^{i}$ is $\left(\begin{array}{cc}0 & A \\ -A^{T} & 0\end{array}\right)$, where $A$ is upper triangular with diagonal entries $\omega\left(x_{i+r}, x_{i+r+n}\right), 1 \leqslant r \leqslant n$.

2.3. Continuous invariants. Given an $(n, N)$-Lagrangian configuration $\left(X_{1}, \ldots, X_{N}\right)$, fix representatives: non-zero points $x_{i}$ on the lines $X_{i}$. As in Section 1.1, extend the $N$-tuple $\left(x_{1}, \ldots, x_{N}\right)$ to an $N$-antiperiodic sequence

$$
\left(x_{i}\right)_{i \in \mathbb{Z}}, \quad x_{i \pm N}:=-x_{i} .
$$

Write $\omega_{i j}$ for $\omega\left(x_{i}, x_{j}\right)$, and observe that

$$
\omega_{i+N, j}=\omega_{j i}=-\omega_{i j}, \quad \omega_{j, i+N}=\omega_{i j} .
$$

Of course the $\omega_{i j}$ depend on the choice of the $x_{i}$, but the symplectic cross-ratios of the configuration do not. Define projective symplectic invariants

$$
c_{i_{1} i_{2} j_{1} j_{2}}:=\left[x_{i_{1}}, x_{i_{2}} ; x_{j_{1}}, x_{j_{2}}\right]=\frac{\omega_{i_{1} j_{1}} \omega_{i_{2} j_{2}}}{\omega_{i_{1} j_{2}} \omega_{i_{2} j_{1}}} .
$$


Note that $c_{i_{1} i_{2} j_{1} j_{2}}$ has the symmetries (2.2) and in addition is invariant under the addition of $N$ to any of the four indices. Due to the Lagrangian condition many of the $c_{i_{1}} i_{2} j_{1} j_{2}$ vanish or are not well defined. To be precise, define the $N$-cyclic distance $|i-j|_{N}$ between any two integers $i$ and $j$ to be their "separation modulo $N^{\prime \prime}$ :

$$
|i-j|_{N}:=\min \{|i-j+q N|: q \in \mathbb{Z}\} .
$$

The Lagrangian condition is $\omega_{i j}=0$ for $|i-j|_{N}<n$. Therefore $c_{i_{1} i_{2} j_{1} j_{2}}$ is either zero or undefined unless $\left|i_{\varepsilon_{i}}-j_{\varepsilon_{j}}\right|_{N} \geqslant n$ for $\varepsilon_{i}$ and $\varepsilon_{j}$ either 1 or 2 . Moreover, if either $i_{1}=i_{2}$ or $j_{1}=j_{2}$, then $c_{i_{1} i_{2} j_{1} j_{2}}$ is 1 if defined. It follows that there are no non-trivial cross-ratios when $N$ is $2 n$ or $2 n+1$, and the only cross-ratios of configurations with $N=2 n+2$ taking values other than 0 and 1 are the $c_{i}$ of (1.4):

$$
c_{i}=c_{i, i+1, i+n+1, i+n+2}=\frac{\omega_{i, i+n+1} \omega_{i+1, i+n+2}}{\omega_{i-n, i} \omega_{i+1, i+n+1}} .
$$

Observe that for $N=2 n+2$ these cross-ratios are $(n+1)$-periodic. Lemma 2.2 shows that in this case they are also always well defined.

We conjecture that in general, (2.5) is a coordinate ring on $\mathcal{L}_{n, N}(\mathbb{K})$ with polynomial relations. This is confirmed only for $N \leqslant 2 n+2$. It would be interesting to find minimal cyclically invariant subsets of (2.5) providing such coordinate rings.

2.4. Opposite configurations and sign invariants. Note that the negation of a symplectic form is also a symplectic form; in particular, $-\omega$ is a symplectic form on $\mathbb{K}^{2 n}$. This leads to the notion of opposite Lagrangian configurations. To be concrete, observe that the $2 n \times 2 n$ matrix $Q=\left(\begin{array}{cc}\text { Id } & 0 \\ 0 & - \text { Id }\end{array}\right)$ has the property $\omega(Q x, Q y)=-\omega(x, y)$ for all $x$ and $y$ in $\mathbb{K}^{2 n}$.

In light of the fact that conjugation by $Q$ preserves $\operatorname{Sp}(2 n, \mathbb{K})$, the following lemma is clear.

Lemma 2.3. (i) If $\left(X_{1}, \ldots, X_{N}\right)$ is a Lagrangian configuration, then so is $\left(Q X_{1}, \ldots, Q X_{N}\right)$. We refer to them as opposites.

(ii) Opposite configurations have the same cross-ratios, and if one is generic, then so is the other.

(iii) If two Lagrangian configurations are equivalent, then their opposites are also equivalent.

Therefore we may speak of opposite equivalence classes in $\mathcal{L}_{n, N}(\mathbb{K})$. Because $Q$ is not symplectic, a configuration is not a priori equivalent to its opposite. In order to resolve the situation we define the sign invariants. Let us write sgn for the sign function:

$$
\operatorname{sgn}: \mathbb{R} \backslash\{0\} \rightarrow\{ \pm 1\} .
$$

Let $\left(\mathbb{R} x_{1}, \ldots, \mathbb{R} x_{N}\right)$ be a real $(n, N)$-Lagrangian configuration, and suppose that $j_{0}, j_{1}, \ldots, j_{r}$ are any integers such that $j_{0} \equiv j_{r}$ modulo $N$. If $\omega_{j_{s-1}, j_{s}} \neq 0$ for all $s$, consider the product $\prod_{1}^{r} \omega_{j_{s-1}, j_{s}}$. Suppose we rescale each $x_{j}$ by some $\lambda_{j}$. Because the $\lambda_{j}$ are by definition $N$-periodic, $\lambda_{j_{0}}=\lambda_{j_{r}}$, so the product rescales by the positive quantity $\prod_{1}^{r} \lambda_{j_{s}}^{2}$. This gives the following lemma.

Lemma 2.4. Let $\left(\mathbb{R} x_{1}, \ldots, \mathbb{R} x_{N}\right)$ be a real $(n, N)$-Lagrangian configuration. If $j_{0}, \ldots, j_{r}$ are integers such that $j_{0} \equiv j_{r}$ modulo $N$ and $\omega_{j_{s-1}, j_{s}} \neq 0$ for all $s$, then $\operatorname{sgn}\left(\prod_{1}^{r} \omega_{j_{s-1}, j_{s}}\right)$ is an invariant of the configuration.

The next proposition elucidates equivalence and inequivalence of opposite configurations. We write GCD for the greatest common divisor function.

Proposition 2.5. $\quad$ (i) Opposite complex configurations are equivalent.

(ii) Opposite real $(n, N)$-configurations are inequivalent if $N / \operatorname{GCD}(n, N)$ is odd.

(iii) Opposite real generic $(n, N)$-configurations are inequivalent for $N>2 n$.

Proof. Let $\left(\mathbb{K} x_{1}, \ldots \mathbb{K} x_{N}\right)$ be a configuration. For (i), note that $i Q$ is symplectic and $i Q \mathbb{C} x_{j}=Q \mathbb{C} x_{j}$.

For (ii), note that the sign invariant $\operatorname{sgn}\left(\prod_{s=1}^{N / \operatorname{GCD}(n, N)} \omega_{(s-1) n, s n}\right)$ negates under passage to the representatives $Q x_{j}$ of the opposite configuration. 
CHARLES H. CONLEY AND VALENTIN OVSIENKO

In the setting of (iii), it is always possible to find a sequence $j_{0}, \ldots, j_{r}$ with $r$ odd, $j_{0} \equiv j_{r}$ modulo $N$, and $\left|j_{s}-j_{s-1}\right|_{N} \geqslant n$, whence the invariant $\operatorname{sgn}\left(\prod_{1}^{r} \omega_{j_{s-1}, j_{s}}\right)$ distinguishes between the configuration and its opposite.

2.5. Dimensions and standard configurations. We now discuss the dimension of $\mathcal{L}_{n, N}$, which determines the number of independent relations which must be satisfied by any coordinate ring of symplectic invariants of configurations. It turns out that for $N \geqslant 2 n+2$, this dimension is always strictly less than the number of non-trivial symplectic cross-ratios. As stated in Section 1.3. at $N=2 n+2$ the dimension is $n$ and there are $n+1$ invariants $c_{i}$, so there must be one relation. It is given in Theorem 1 .

Let us begin by defining a convenient normal form for sets of representatives of configurations. Recall that we write $\left\{e_{1}, \ldots, e_{n}, f_{1}, \ldots, f_{n}\right\}$ for the standard basis of $\mathbb{K}^{2 n}$.

Definition. Given an $(n, N)$-Lagrangian configuration $\left(\mathbb{K} x_{1}, \ldots, \mathbb{K} x_{N}\right)$, the representatives $x_{1}, \ldots, x_{N}$ are said to be standard if for some vectors $g_{i} \in\left\langle f_{1}, \ldots, f_{i-1}\right\rangle, x_{1}, \ldots, x_{2 n}$ are given by

$$
x_{1}=e_{1}, \quad x_{2}=e_{2}, \quad \ldots, \quad x_{n}=e_{n}, \quad x_{n+1}=f_{1}, \quad x_{n+2}=f_{2}+g_{2}, \quad \ldots, \quad x_{2 n}=f_{n}+g_{n} .
$$

Lemma 2.6. $\quad$ (i) Every $(n, N)$-Lagrangian configuration is symplectically equivalent to a configuration with standard representatives.

(ii) In a configuration with standard representatives, $\omega\left(e_{j}, g_{i}\right)=0$ for $j<i+2 n-N$.

(iii) In a generic configuration with standard representatives, $\omega\left(e_{j}, g_{i}\right) \neq 0$ for $i+2 n-N \leqslant j<i$.

Proof. Given an $(n, N)$-configuration $\left(\mathbb{K} x_{1}, \ldots, \mathbb{K} x_{N}\right)$, consider the Gram matrix $\Omega\left(x_{1}, \ldots, x_{2 n}\right)$. Because it has the form noted in the proof of Lemma 2.2. Lemma 2.1(iii) shows that there is a symplectic transformation mapping $x_{i}$ to $e_{i}$ and $x_{n+i}$ to a multiple of $f_{i}+g_{i}$ for $1 \leqslant i \leqslant n, g_{1}$ being zero and $g_{2}, \ldots, g_{n}$ having the desired form. To complete the proof, rescale the representatives. For (ii) and (iii), refer to the definitions of Lagrangian and generic.

Proposition 2.7. For $N>2 n$, the variety of $(n, N)$-Lagrangian configurations is $n N$-dimensional. $\operatorname{Sp}(2 n, \mathbb{K})$ acts freely on generic configurations, and so $\mathcal{L}_{n, N}(\mathbb{K})$ is $n(N-2 n-1)$-dimensional.

Proof. Consider the process of constructing an $(n, N)$-configuration by choosing first $X_{1}$, then $X_{2}$, and so on to $X_{N}$. Count the number of degrees of freedom available in choosing each $X_{i}$ as follows. There are $2 n-1$ degrees of freedom for $X_{1}$, as it is simply an arbitrary line. There are $2 n-2$ degrees of freedom for $X_{2}$, as $\left\langle X_{1}, X_{2}\right\rangle$ must be isotropic, and $2 n-3$ for $X_{3}$, as $\left\langle X_{1}, X_{2}, X_{3}\right\rangle$ must be isotropic. Continuing, for $i \leqslant n$ we find that there are $2 n-i$ degrees for $X_{i}$. For $n \leqslant i \leqslant N-n+1$ there are $n$ degrees for $X_{i}$, as the only constraints arise from the requirement that $\left\langle X_{i-n+1}, \ldots, X_{i}\right\rangle$ be isotropic.

There are $n-1$ degrees for $X_{N-n+2}$, as in addition to the above isotropy requirement, $\left\langle X_{N-n+2}, X_{1}\right\rangle$ must be isotropic. Continuing, for $N-n+1 \leqslant i \leqslant N$ there are $N+1-i$ degrees for $X_{i}$. In particular, all of the $X_{i}$ can be chosen, and there are a total of $n N$ degrees of freedom in choosing the configuration.

To complete the proof, it suffices to prove that any symplectic transformation $T$ stabilizing a generic standard configuration $\left(\mathbb{K} x_{1}, \ldots, \mathbb{K} x_{N}\right)$ is the identitity Id. Keeping in mind that $T$ must stabilize $\mathbb{K} x_{i}$ but not necessarily $x_{i}$, we find that it must be of the form $\left(\begin{array}{cc}D & 0 \\ 0 & D^{-1}\end{array}\right)$ for some diagonal matrix $D$. Because $N>2 n, \omega\left(e_{i-1}, g_{i}\right) \neq 0$, forcing $D$ to be scalar. Finally, genericity implies that $\omega\left(e_{n}, x_{2 n+1}\right)$ and $\omega\left(f_{1}, x_{2 n+1}\right)$ are both non-zero. Hence the condition that $T$ stabilize $\mathbb{K} x_{2 n+1}$ forces $D=\operatorname{Id}$.

Corollary 2.8. $\mathcal{L}_{n, N}(\mathbb{R})$ and $\mathcal{L}_{n, N}(\mathbb{C})$ are smooth manifolds.

2.6. The case $N>2 n+2$. We conclude this section with a few remarks on configurations with $N>$ $2 n+2$. We begin with a corollary of Lemma 2.2. Let us single out the symplectic cross-ratios

$$
\gamma_{i j}:=c_{i, j+n, j, i+n}=\frac{\omega_{i j} \omega_{i+n, j+n}}{\omega_{i, i+n} \omega_{j, j+n}}
$$


Corollary 2.9. Let $\left(\mathbb{K} x_{1}, \ldots, \mathbb{K} x_{N}\right)$ be an $(n, N)$-Lagrangian configuration. Then $\gamma_{i j}$ is defined for all $i$ and $j$, and it is 0 for $|i-j|_{N}<n$ and 1 for $|i-j|_{N}=n$. The configuration is generic if and only if $\gamma_{i j}$ is non-zero whenever $|i-j|_{N} \geqslant n$.

Observe that $\gamma_{i+N, j}=\gamma_{j i}=\gamma_{i j}$. For $N=2 n+2$, all non-trivial symplectic cross-ratios are $\gamma_{i j}$ 's, as $c_{i}=\gamma_{i+1, i-n}$. This is not true for $N>2 n+2$ : for example, for $(2,7)$-configurations, $c_{1,2,4,5}$ is not a $\gamma_{i j}$. However, we do have the relation

$$
c_{i_{1} i_{2} j_{1} j_{2}} c_{i_{1}+n, i_{2}+n, j_{1}+n, j_{2}+n}=\frac{\gamma_{i_{1} j_{1}} \gamma_{i_{2} j_{2}}}{\gamma_{i_{1} j_{2}} \gamma_{i_{2} j_{1}}} .
$$

\section{The MAIN RESUlts}

In this section we describe the moduli space $\mathcal{L}_{n, N}(\mathbb{K})$ of generic $(n, N)$-configurations over $\mathbb{K}=\mathbb{R}$ or $\mathbb{C}$ for $N$ equal to $2 n, 2 n+1$, and $2 n+2$. In the first two cases it is trivial and is described in Proposition 3.1. Let us mention that a related result is proven in [15, for three lines in $\mathbb{K}^{2 n}$.

The first non-trivial case, $\mathcal{L}_{n, 2 n+2}(\mathbb{K})$, is described in Theorem 1, there are $n+1$ cross-ratios, which satisfy a single relation. In the case that $n$ is even and $\mathbb{K}=\mathbb{C}$, Theorem 2 provides a combinatorial interpretation of this relation.

\subsection{The cases $N=2 n$ and $N=2 n+1$.}

Proposition 3.1. $\quad$ (i) For $N=2 n$ or $2 n+1$, all $(n, N)$-Lagrangian configurations are generic.

(ii) $\mathcal{L}_{n, 2 n}(\mathbb{K})$ is a single point for $\mathbb{K}=\mathbb{R}$ or $\mathbb{C}$.

(iii) $\mathcal{L}_{n, 2 n+1}(\mathbb{R})$ consists of two opposite points, and $\mathcal{L}_{n, 2 n+1}(\mathbb{C})$ is a single point.

Proof. Part (i) is clear from Lemma 2.2 and the fact that $|i-j|_{2 n+1}$ never exceeds $n$. For (ii), Lemma 2.6 shows that all configurations are equivalent to $\left(\mathbb{K} e_{1}, \ldots, \mathbb{K} e_{n}, \mathbb{K} f_{1}, \ldots, \mathbb{K} f_{n}\right)$.

For (iii), Lemma 2.6] shows that any configuration is equivalent to some $\left(\mathbb{K} x_{1}, \ldots, \mathbb{K} x_{2 n+1}\right)$ with $x_{i}=e_{i}$ for $1 \leqslant i \leqslant n, x_{n+1}=f_{1}$, and $x_{n+i}=f_{i}+b_{i} f_{i-1}$ for $2 \leqslant i \leqslant n$, where the $b_{i}$ are non-zero scalars. Define $b_{1}:=1$, apply the diagonal symplectic transformation $e_{i} \mapsto e_{i} \prod_{1}^{i} b_{j}^{-1}$ and $f_{i} \mapsto f_{i} \prod_{1}^{i} b_{j}$, and rescale to deduce that we may assume $b_{i}=1$ for all $i$.

Combine the Lagrangian condition with genericity to see that $x_{2 n+1}$ can be rescaled to take the form $f_{n}+b_{0} \sum_{1}^{n}(-1)^{i} e_{i}$ for some scalar $b_{0} \neq 0$. For $\mathbb{K}=\mathbb{R}$ and $b_{0}>0$ or $\mathbb{K}=\mathbb{C}$, apply the symplectic transformation $e_{i} \mapsto b_{0}^{1 / 2} e_{i}$ and $f_{i} \mapsto b_{0}^{-1 / 2} f_{i}$ and rescale to arrive at $x_{2 n+1}=f_{n}+\sum_{1}^{n}(-1)^{i} e_{i}$. For $\mathbb{K}=\mathbb{R}$ and $b_{0}<0$, use $\left(-b_{0}\right)^{1 / 2}$ in place of $b_{0}^{1 / 2}$ to arrive at $x_{2 n+1}=f_{n}-\sum_{1}^{n}(-1)^{i} e_{i}$. By Proposition 2.5, the two signs of $b_{0}$ give opposite real equivalence classes.

Combining this argument with Section 2.4 yields the following corollary. For $\varepsilon= \pm 1$, consider the $(n, 2 n+1)$-configurations with representatives

$$
\left(e_{1}, e_{2}, \ldots, e_{n}, \quad \varepsilon f_{1}, \varepsilon\left(f_{1}+f_{2}\right), \varepsilon\left(f_{2}+f_{3}\right), \ldots, \varepsilon\left(f_{n-1}+f_{n}\right), \quad \varepsilon f_{n}+\sum_{i}^{n}(-1)^{i} e_{i}\right) .
$$

Observe that these representatives have $\omega_{i, n+i}=\varepsilon$ for all $i$.

Corollary 3.2. $\quad$ (i) The unique element of $\mathcal{L}_{n, 2 n+1}(\mathbb{C})$ is the class of (3.1) for $\varepsilon=1$.

(ii) The two opposite elements of $\mathcal{L}_{n, 2 n+1}(\mathbb{R})$ are the classes of (3.1) for $\varepsilon= \pm 1$.

(iii) An arbitrary $(n, 2 n+1)$-Lagrangian configuration $\left(\mathbb{R} x_{1}, \ldots, \mathbb{R} x_{2 n+1}\right)$ over $\mathbb{R}$ is equivalent to (3.1) with $\varepsilon=\operatorname{sgn}\left(\prod_{i=0}^{2 n} \omega_{i, n+i}\right)$. 
3.2. The first non-trivial case: $N=2 n+2$. We now give the description of $\mathcal{L}_{n, 2 n+2}(\mathbb{K})$, one of our main results. Recall that the symplectic cross-ratios $c_{i}$ given in (1.4) and (2.6) are $(n+1)$-periodic, are the only non-trivial cross-ratios on $(n, 2 n+2)$-configurations, and are non-zero on generic configurations.

Let us use the standard notation $\lfloor x \mid$ for the integer part of a real number $x$. In order to state the result, we define an index set $\mathcal{I}_{n}(r) \subset\{0, \ldots, n\}^{r}$ for $1 \leqslant r \leqslant\lfloor(n+1) / 2\rfloor$ :

$$
\mathcal{I}_{n}(r):=\left\{\left(i_{1}, \ldots, i_{r}\right): 0 \leqslant i_{1}, i_{s}+1<i_{s+1} \text { for } 1 \leqslant s<r, i_{r} \leqslant n \text {, and if } i_{1}=0 \text {, then } i_{r}<n\right\} .
$$

Observe that this definition may be rephrased as follows: $\mathcal{I}_{n}(r)$ is the set of strictly increasing $r$-tuples $0 \leqslant i_{1}<\cdots<i_{r} \leqslant n$ such that the $(n+1)$-cyclic distances $\left|i_{s}-i_{s^{\prime}}\right|_{n+1}$ exceed 1 for all $s \neq s^{\prime}$. Let us give the initial cases explicitly:

- For $n \geqslant 1, \mathcal{I}_{n}(1)=\{0,1, \ldots, n\}$.

- For $n \geqslant 3, \mathcal{I}_{n}(2)=\{(0,2),(0,3), \ldots,(0, n-1) ;(1,3), \ldots,(1, n) ; \ldots ;(n-2, n)\}$.

- $\mathcal{I}_{5}(3)=\{(0,2,4),(1,3,5)\}$, and $\mathcal{I}_{6}(3)=\{(0,2,4),(0,2,5),(0,3,5),(1,3,5),(1,3,6),(1,4,6)\}$.

Theorem 1. (i) On $\mathcal{L}_{n, 2 n+2}(\mathbb{K})$, the $n+1$ symplectic cross-ratios $c_{0}, \ldots, c_{n}$ satisfy the relation

$$
0=1+\sum_{r=1}^{\lfloor(n+1) / 2\rfloor} \sum_{\mathcal{I}_{n}(r)} \frac{(-1)^{r}}{c_{i_{1}} c_{i_{2}} \cdots c_{i_{r}}} .
$$

(ii) $\left(c_{0}, \ldots, c_{n}\right)$ is a coordinate ring on $\mathcal{L}_{n, 2 n+2}(\mathbb{K})$ : any two generic $(n, 2 n+2)$-equivalence classes with the same cross-ratios are equal for $\mathbb{K}=\mathbb{C}$, and either equal or opposite for $\mathbb{K}=\mathbb{R}$.

(iii) (3.2) is the only relation on the cross-ratios: if $c_{0}, \ldots, c_{n}$ are arbitrary non-zero scalars in $\mathbb{K}$ satisfying (3.2), then they are the cross-ratios of some generic $(n, 2 n+2)$-configuration over $\mathbb{K}$.

Thus $\mathcal{L}_{n, 2 n+2}(\mathbb{K})$ may be viewed as a dense open subset of the algebraic hypersurface in $\mathbb{K}^{n+1}$ defined by multiplying (3.2) by $c_{0} \cdots c_{n}$. We prove Theorem 1 in Section 4

Examples. (a) For $\mathcal{L}_{1,4}(\mathbb{K})$, the relation on the two cross-ratios of quadrilaterals in $\mathbb{K} \mathbb{P}^{1}$ simply relates two forms of the classical cross-ratio:

$$
\frac{1}{c_{0}}+\frac{1}{c_{1}}=1 .
$$

(b) For $\mathcal{L}_{2,6}(\mathbb{K})$, the relation on the three cross-ratios $c_{0}, c_{1}$, and $c_{2}$ of Legendrian hexagons in $\mathbb{K} \mathbb{P}^{3}$ was given in (1.1).

(c) The moduli space $\mathcal{L}_{3,8}(\mathbb{K})$ of Legendrian octagons in $\mathbb{K} \mathbb{P}^{5}$ is parametrized by the four cross-ratios $c_{0}, c_{1}, c_{2}$, and $c_{3}$, subject to the relation

$$
\frac{1}{c_{0}}+\frac{1}{c_{1}}+\frac{1}{c_{2}}+\frac{1}{c_{3}}-\frac{1}{c_{0} c_{2}}-\frac{1}{c_{1} c_{3}}=1 .
$$

(d) For $\mathcal{L}_{4,10}(\mathbb{K})$, the Legendrian decagons in $\mathbb{K}^{7}$, the five cross-ratios $c_{0}, \ldots, c_{4}$ satisfy

$$
\frac{1}{c_{0}}+\frac{1}{c_{1}}+\frac{1}{c_{2}}+\frac{1}{c_{3}}+\frac{1}{c_{4}}-\frac{1}{c_{0} c_{2}}-\frac{1}{c_{1} c_{3}}-\frac{1}{c_{2} c_{4}}-\frac{1}{c_{3} c_{0}}-\frac{1}{c_{4} c_{1}}=1 .
$$

The following analog of Figure 1 depicts $c_{0}$ in this setting:

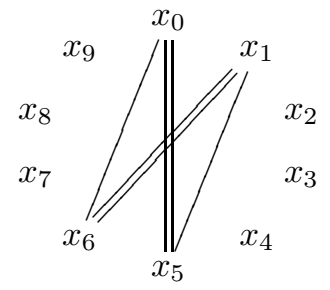

Figure 2. The cross-ratio $c_{0}=\frac{\omega_{0,5} \omega_{1,6}}{\omega_{0,6} \omega_{1,5}}$ on $\mathcal{L}_{4,10}$. 
3.3. The cyclic continuant. In Section 5 we will describe certain normalized choices of representatives of $(n, 2 n+2)$-Lagrangian configurations. The nature of the normalizations depends on the field $\mathbb{K}$ and the parity of $n$. The situation is simplest for $n$ even and $\mathbb{K}=\mathbb{C}$, the case generalizing the hexagonal example in Section 1.1 At this point we state the relevant normalization, Proposition 3.3, along with the corresponding specialization of Theorem 10. Theorem 2 A notable feature of this specialization is that the relation (3.2) is expressed in terms of continuants. The proofs will be given in Section 5.1

The classical continuant is the tridiagonal determinant

$$
K_{n}\left(a_{1}, \ldots, a_{n}\right):=\left|\begin{array}{ccccc}
a_{1} & 1 & & & \\
1 & a_{2} & 1 & & \\
& \ddots & \ddots & \ddots & \\
& & 1 & a_{n-1} & 1 \\
& & & 1 & a_{n}
\end{array}\right| .
$$

This remarkable polynomial has a long history. It arises in the theory of continued fractions and many other areas; see for example $[3$ and references therein.

The main topic of 3 is the cyclic continuant, defined as

$$
R_{n+1}\left(a_{0}, \ldots, a_{n}\right):=K_{n+1}\left(a_{0}, \ldots, a_{n}\right)-K_{n-1}\left(a_{1}, \ldots, a_{n-1}\right) .
$$

It too is related to continued fractions, via the identity

$$
R_{n+1}\left(a_{0}, \ldots, a_{n}\right)=\operatorname{tr}\left(\begin{array}{cc}
a_{n} & -1 \\
1 & 0
\end{array}\right)\left(\begin{array}{cc}
a_{n-1} & -1 \\
1 & 0
\end{array}\right) \cdots\left(\begin{array}{cc}
a_{0} & -1 \\
1 & 0
\end{array}\right) .
$$

As an illustration, let us display $R_{5}\left(a_{0}, a_{1}, a_{2}, a_{3}, a_{4}\right)$ :

$$
a_{0} a_{1} a_{2} a_{3} a_{4}-\left(a_{0} a_{1} a_{2}+a_{1} a_{2} a_{3}+a_{2} a_{3} a_{4}+a_{3} a_{4} a_{0}+a_{4} a_{0} a_{1}\right)+\left(a_{0}+a_{1}+a_{2}+a_{3}+a_{4}\right) .
$$

In the next two statements, let $\left(X_{0}, \ldots, X_{2 n+1}\right)$ be a generic $(n, 2 n+2)$-Lagrangian configuration over $\mathbb{K}$ with representatives $x_{0}, \ldots, x_{2 n+1}$, symplectic products $\omega_{i j}:=\omega\left(x_{i}, x_{j}\right)$, and cross-ratios $c_{0}, \ldots, c_{n}$. Recall from Section 1.3 that we refer to the $(2 n+2)$-periodic sequence $\left(\omega_{i, i+n}\right)_{i}$ as the set of symplectic subdiameters of $\left(x_{i}\right)_{i}$, and the $(n+1)$-periodic sequence $\left(\omega_{i, i+n+1}\right)_{i}$ as the set of symplectic diameters.

Proposition 3.3. For $n$ even and $\mathbb{K}=\mathbb{C},\left(X_{0}, \ldots, X_{2 n+1}\right)$ admits exactly four choices of representatives whose symplectic subdiameters are all 1 . Fix such a choice, $x_{0}, \ldots, x_{2 n+1}$, and set $a_{i}:=\omega_{i, i+n+1}$.

(i) The four choices are $\left(x_{i}\right)_{i},\left(-x_{i}\right)_{i},\left((-1)^{i} x_{i}\right)_{i}$, and $\left(-(-1)^{i} x_{i}\right)_{i}$.

(ii) The first two choices in (i) have symplectic diameters $\left(a_{i}\right)_{i}$, and the second two have symplectic diameters $\left(-a_{i}\right)_{i}$.

(iii) The cross-ratios of the configuration are $c_{i}=a_{i} a_{i+1}$. The symplectic diameters satisfy

$$
a_{i}^{2}=\frac{c_{i} c_{i+2} \cdots c_{i+n}}{c_{i+1} c_{i+3} \cdots c_{i+n-1}} .
$$

This result shows that the symplectic diameters of the choices of representatives whose symplectic subdiameters are all 1 are invariants of the configuration, defined up to an overall sign. We refer to them as the normalized symplectic diameters and denote them by $\pm\left(a_{0}, \ldots, a_{n}\right)$.

In reading the next result, keep in mind that $K_{n}$ and hence also $R_{n}$ are of parity $(-1)^{n}$ under negation of all their arguments, so $R_{n+1}\left(a_{0}, \ldots, a_{n}\right)$ vanishes if and only if $R_{n+1}\left(-a_{0}, \ldots,-a_{n}\right)$ vanishes.

Theorem 2. Let $n$ be even.

(i) The cyclic continuant of the normalized symplectic diameters on $\mathcal{L}_{n, 2 n+2}(\mathbb{C})$ vanishes:

$$
R_{n+1}\left(a_{0}, \ldots, a_{n}\right)=0 .
$$

(ii) Equivalence classes in $\mathcal{L}_{n, 2 n+2}(\mathbb{C})$ with the same normalized symplectic diameters are equal. 
(iii) If $\pm\left(a_{0}, \ldots, a_{n}\right)$ are arbitrary non-zero complex scalars with cyclic continuant zero, then they are the normalized symplectic diameters of some equivalence class in $\mathcal{L}_{n, 2 n+2}(\mathbb{C})$.

As noted above, Proposition 3.3 and Theorem 2 are proven in Section 5.1

\section{Pfaffians and the proof of Theorem 1}

This section contains the proof of our main result, Theorem 1 .

4.1. Tridiagonal determinants and the proof of Theorem 1 (i). The following formula is well-known and easily proven by induction.

Proposition 4.1. The tridiagonal matrix

$$
A_{n}:=\left(\begin{array}{ccccc}
a_{11} & a_{12} & & & \\
a_{21} & a_{22} & a_{23} & & \\
& \ddots & \ddots & \ddots & \\
& & a_{n-1, n-2} & a_{n-1, n-1} & a_{n-1, n} \\
& & & a_{n, n-1} & a_{n n}
\end{array}\right)
$$

has determinant

$$
\operatorname{det}\left(A_{n}\right)=\left(\prod_{k=1}^{n} a_{k k}\right) \sum_{r=0}^{\lfloor n / 2\rfloor}(-1)^{r} \sum_{\substack{1 \leqslant i_{1}<\cdots<i_{r}<n, i_{s}+1<i_{s+1} \forall \forall \leqslant s<r}} \prod_{s=1}^{r} \frac{a_{i_{s}, i_{s}+1} a_{i_{s}+1, i_{s}}}{a_{i_{s} i_{s}} a_{i_{s}+1, i_{s}+1}},
$$

where the summand at $r=0$ is understood to be 1.

Remark. When the sub- and superdiagonal entries of $A_{n}$ are all 1, its determinant is in fact the continuant $K_{n}$. In this case Euler discovered a pleasing interpretation of Proposition 4.1, which generalizes as follows: to write out all summands of (4.2), start with $a_{11} \cdots a_{n n}$, and for each set of disjoint adjacent pairs $(i, i+1), 1 \leqslant i<n$, replace $a_{i i} a_{i+1, i+1}$ by $-a_{i, i+1} a_{i+1, i}$. We refer to this process as Euler's replacement algorithm.

Examples. Indicating pairs by parentheses, $\operatorname{det}\left(A_{3}\right), \operatorname{det}\left(A_{4}\right)$, and $\operatorname{det}\left(A_{5}\right)$ are, respectively,

- $a_{11} a_{22} a_{33}-\left(a_{12} a_{21}\right) a_{33}-a_{11}\left(a_{23} a_{32}\right)$,

- $a_{11} a_{22} a_{33} a_{44}-\left(a_{12} a_{21}\right) a_{33} a_{44}-a_{11}\left(a_{23} a_{32}\right) a_{44}-a_{11} a_{22}\left(a_{34} a_{43}\right)+\left(a_{12} a_{21}\right)\left(a_{34} a_{43}\right)$,

- $a_{11} a_{22} a_{33} a_{44} a_{55}-\left(a_{12} a_{21}\right) a_{33} a_{44} a_{55}-a_{11}\left(a_{23} a_{32}\right) a_{44} a_{55}-a_{11} a_{22}\left(a_{34} a_{43}\right) a_{55}-a_{11} a_{22} a_{33}\left(a_{45} a_{54}\right)$

$$
+\left(a_{12} a_{21}\right)\left(a_{34} a_{43}\right) a_{55}+\left(a_{12} a_{21}\right) a_{33}\left(a_{45} a_{54}\right)+a_{11}\left(a_{23} a_{32}\right)\left(a_{45} a_{54}\right) .
$$

Recall now that the Pfaffian $\operatorname{pf}(S)$ is a polynomial in the entries of a skew-symmetric matrix $S$ whose square is $\operatorname{det}(S)$. Consider the $(2 n+2) \times(2 n+2)$ matrix

$$
\Omega_{n}:=\left(\begin{array}{cc}
\omega_{0, n} E & A \\
-A^{T} & \omega_{n+1,2 n+1} E
\end{array}\right)
$$


with ingredients defined as follows: $E$ is the skew-symmetric $(n+1) \times(n+1)$ matrix $e_{1, n+1}-e_{n+1,1}\left(e_{i j}\right.$ being the elementary matrix whose $i j^{\text {th }}$ entry is 1 and whose other entries are 0$), A$ is given by

$$
A=\left(\begin{array}{ccccc}
\omega_{0, n+1} & \omega_{0, n+2} & & & \\
\omega_{1, n+1} & \omega_{1, n+2} & \omega_{1, n+3} & & \\
& \ddots & \ddots & \ddots & \\
& & \omega_{n-1,2 n-1} & \omega_{n-1,2 n} & \omega_{n-1,2 n+1} \\
& & & \omega_{n, 2 n} & \omega_{n, 2 n+1}
\end{array}\right),
$$

and the $\omega_{i j}$ are arbitrary scalars. As a visual aid, we illustrate $\Omega_{n}$ in long form:

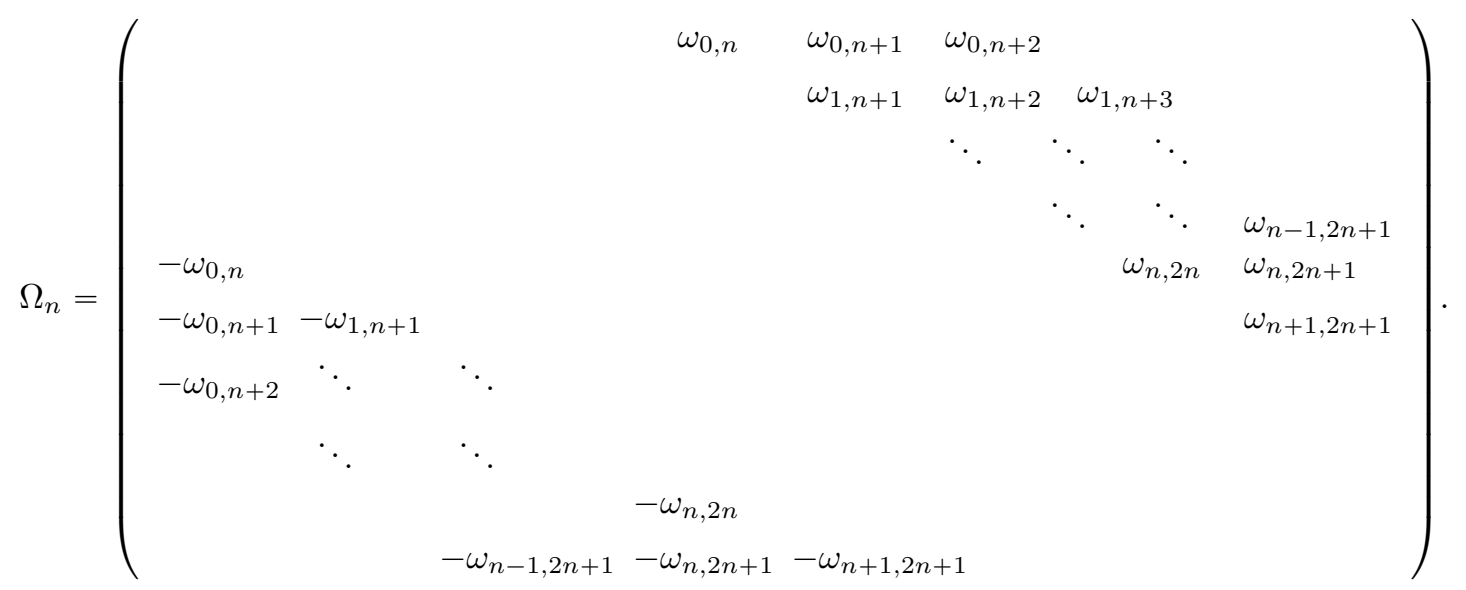

Proposition 4.2. Recall $\mathcal{I}_{n}(r)$ from Section 3.2. The Pfaffian $\operatorname{pf}\left(\Omega_{n}\right)$ is given by the expression

$$
(-1)^{n(n+1) / 2}\left(\prod_{k=0}^{n} \omega_{k, k+n+1}\right) \sum_{r=0}^{\lfloor(n+1) / 2\rfloor}(-1)^{r} \sum_{\mathcal{I}_{n}(r)} \prod_{s=1}^{r} \frac{\omega_{i_{s}, i_{s}+n+2} \omega_{i_{s}+1, i_{s}+n+1}}{\omega_{i_{s}, i_{s}+n+1} \omega_{i_{s}+1, i_{s}+n+2}},
$$

where the summand at $r=0$ is taken to be 1 , and whenever $\omega_{i, 2 n+2}$ appears it should be replaced by $\omega_{0, i}$.

Proof. Given any $m \times m$ matrix $A$, let $A_{\text {mid }}$ be the $(m-2) \times(m-2)$ matrix obtained by deleting the top and bottom rows and left and right columns of $A$. The following lemma is proven in 3 .

Lemma 4.3. For any $m \times m$ matrix $A$ and any scalars $x$ and $y$, one has

$$
\operatorname{pf}\left(\begin{array}{cc}
x E & A \\
-A^{T} & y E
\end{array}\right)=(-1)^{m(m-1) / 2}\left(\operatorname{det}(A)-x y \operatorname{det}\left(A_{\text {mid }}\right)\right) .
$$

Applying both (4.2) and (4.7) to (4.3) yields (4.6).

Remark. The Pfaffian (4.6) may be interpreted via a cyclic version of Euler's replacement algorithm, the "cyclic replacement algorithm": to write out all summands of $(-1)^{n(n+1) / 2} \operatorname{pf}\left(\Omega_{n}\right)$, start with

$$
\omega_{0, n+1} \omega_{1, n+2} \cdots \omega_{n, 2 n+1},
$$

and for each set of disjoint cyclically adjacent pairs $(i, i+1), 0 \leqslant i \leqslant n$, replace $\omega_{i, i+n+1} \omega_{i+1, i+n+2}$ by $-\omega_{i, i+n+2} \omega_{i+1, i+n+1}$. Cyclically adjacent indicates that the pair $(n, n+1)$ is read as $(n, 0)$. When it is in the set of pairs, replace $\omega_{0, n+1} \omega_{n, 2 n+1}$ by $-\omega_{0, n} \omega_{n+1,2 n+1}$. To explain, note that by the replacement rule $\omega_{i, 2 n+2}=\omega_{0, i}$,

$$
\omega_{0, n+1} \omega_{n, 2 n+1}=\omega_{n, 2 n+1} \omega_{n+1,2 n+2}, \quad \omega_{n, 2 n+2} \omega_{n+1,2 n+1}=\omega_{0, n} \omega_{n+1,2 n+1} .
$$


Examples. Let us give (4.6) explicitly for small $n$. As in the examples below Proposition 4.1, we indicate pairs with parentheses. By (4.7) and (4.4), the summands from sets not containing the special pair $(n, 0)$ add up to an $(n+1) \times(n+1)$ tridiagonal determinant, and the summands from sets containing it add up to $\omega_{0, n} \omega_{n+1,2 n+1}$ times an $(n-1) \times(n-1)$ tridiagonal determinant. To emphasize this, we have separated the two types of terms with square brackets and factored $\omega_{0, n} \omega_{n+1,2 n+1}$ out of the second type.

Note that at $n=1$ there are two ways to delete the lone pair $(0,1)$ : as $(0,1)$, or as $(1,0)$. For $n=1$, 2 , and $3,(-1)^{n(n+1) / 2} \operatorname{pf}\left(\Omega_{n}\right)$ is, respectively,

$$
\begin{aligned}
& -\left[\omega_{02} \omega_{13}-\left(\omega_{03} \omega_{12}\right)\right]-\left(\omega_{01} \omega_{23}\right)[1] \\
\text { - } & {\left[\omega_{03} \omega_{14} \omega_{25}-\left(\omega_{04} \omega_{13}\right) \omega_{25}-\omega_{03}\left(\omega_{15} \omega_{24}\right)\right]-\left(\omega_{02} \omega_{35}\right)\left[\omega_{14}\right], } \\
\text { - } & {\left[\omega_{04} \omega_{15} \omega_{26} \omega_{37}-\left(\omega_{05} \omega_{14}\right) \omega_{26} \omega_{37}-\omega_{04}\left(\omega_{16} \omega_{25}\right) \omega_{37}-\omega_{04} \omega_{15}\left(\omega_{27} \omega_{36}\right)+\left(\omega_{05} \omega_{14}\right)\left(\omega_{27} \omega_{36}\right)\right] } \\
- & \left(\omega_{03} \omega_{47}\right)\left[\omega_{15} \omega_{26}-\left(\omega_{16} \omega_{25}\right)\right] .
\end{aligned}
$$

Observe that dividing by $\omega_{0, n+1} \omega_{1, n+2} \cdots \omega_{n, 2 n+1}$ leads to the examples in Section 3.2. and compare the terms in square brackets to the examples below Proposition 4.1 .

Proof of (3.2) . Suppose now that $\left(\mathbb{K} x_{0}, \ldots, \mathbb{K} x_{2 n+1}\right)$ is an $(n, 2 n+2)$-Lagrangian configuration, and revert to our customary notation $\omega_{i j}=\omega\left(x_{i}, x_{j}\right)$. By the Lagrangian condition, the $\omega$-Gram matrix $\Omega\left(x_{0}, \ldots, x_{2 n+1}\right)$ is precisely the matrix $\Omega_{n}$ in (4.3), and by Lemma 2.1(ii),

$$
\operatorname{det}\left(\Omega\left(x_{0}, \ldots, x_{2 n+1}\right)\right)=0 .
$$

Hence Proposition 4.2 yields the following corollary.

Corollary 4.4. Let $\left(\mathbb{K} x_{0}, \ldots, \mathbb{K} x_{2 n+1}\right)$ be an $(n, 2 n+2)$-Lagrangian configuration. Then

$$
0=\left(\prod_{k=0}^{n} \omega_{k, k+n+1}\right) \sum_{r=0}^{\lfloor(n+1) / 2\rfloor}(-1)^{r} \sum_{\mathcal{I}_{n}(r)} \prod_{s=1}^{r} \frac{1}{c_{i_{s}}},
$$

where as usual, the summand at $r=0$ is understood to be 1.

This in turn yields (3.2) of Theorem 1(i), because generic $(n, 2 n+2)$-configurations have $\omega_{k, k+n+1} \neq 0$. Note that (4.6) is polynomial in the $\omega_{i j}$, so after cancellation, Corollary 4.4 gives a non-trivial relation even on non-generic configurations.

4.2. Proof of Theorem 1 (ii). Suppose that $\left(\mathbb{K} x_{1}, \ldots, \mathbb{K} x_{2 n+2}\right)$ and $\left(\mathbb{K} \tilde{x}_{1}, \ldots, \mathbb{K} \tilde{x}_{2 n+2}\right)$ are two generic $(n, 2 n+2)$-Lagrangian configurations with the same cross-ratios $c_{1}, \ldots, c_{n+1}$. Following our convention $\omega_{i j}:=\omega\left(x_{i}, x_{j}\right)$, we set $\tilde{\omega}_{i j}:=\tilde{\omega}\left(x_{i}, x_{j}\right)$.

By Lemma 2.1(iii), in order to prove the two configurations equivalent it suffices to find a renormalization $x_{i} \mapsto \lambda_{i} x_{i}$ such that $\lambda_{i} \lambda_{j} \omega_{i j}=\tilde{\omega}_{i j}$ for all $i$ and $j$. By the Lagrangian condition, we need only do this for $j=i+n$ and $i+n+1$. The argument depends on the parity of $n$.

The case of $n$ even. It is important to keep in mind that here $\operatorname{GCD}(n, 2 n+2)=2$. Hence the subset of lines in an $(n, 2 n+2)$-configuration whose indices have a given parity may be written in either of the following ways:

$$
\left\{\mathbb{K} x_{i}, \mathbb{K} x_{i+2}, \mathbb{K} x_{i+4}, \ldots, \mathbb{K} x_{i+2 n}\right\}=\left\{\mathbb{K} x_{i}, \mathbb{K} x_{i+n}, \mathbb{K} x_{i+2 n}, \ldots, \mathbb{K} x_{i+n^{2}}\right\} .
$$

We begin with the case $\mathbb{K}=\mathbb{C}$. For $i=1, \ldots, 2 n+2$, fix a square root $\chi_{i}$ of $\tilde{\omega}_{i, i+n} / \omega_{i, i+n}$. Extend $(2 n+2)$-periodically to define $\chi_{i}$ for $i \in \mathbb{Z}$. Set

$$
\lambda_{i}:=\frac{\chi_{i} \chi_{i+2 n} \chi_{i+4 n} \cdots \chi_{i+n^{2}}}{\chi_{i+n} \chi_{i+3 n} \cdots \chi_{i+(n-1) n}}=\prod_{r=0}^{n} \chi_{i+r n}^{(-1)^{r}}
$$


and check that $\lambda_{i} \lambda_{i+n}=\tilde{\omega}_{i, i+n} / \omega_{i, i+n}$ for all $i$.

Replacing $x_{i}$ by $\lambda_{i} x_{i}$, we may assume $\omega_{i, i+n}=\tilde{\omega}_{i, i+n}$ for all $i$. Write $\rho_{i}$ for the ratio $\tilde{\omega}_{i, i+n+1} / \omega_{i, i+n+1}$, which is $(n+1)$-periodic. Use the fact that the configurations have the same cross-ratios to obtain $\rho_{i} \rho_{i+1}=1$ for all $i$. Hence

$$
1=\frac{\left(\rho_{i} \rho_{i+1}\right)\left(\rho_{i+2} \rho_{i+3}\right) \cdots\left(\rho_{i+n} \rho_{i+n+1}\right)}{\left(\rho_{i+1} \rho_{i+2}\right)\left(\rho_{i+3} \rho_{i+4}\right) \cdots\left(\rho_{i+n-1} \rho_{i+n}\right)}=\rho_{i}^{2} .
$$

Deduce that the $\rho_{i}$ are either all 1 or all -1 . In the former case we are done. In the latter case, rescale again, replacing $x_{i}$ by $(-1)^{i} x_{i}$. This leaves the $\omega_{i, i+n}$ unchanged and negates the $\omega_{i, i+n+1}$, so again we are done.

Example. For $n=2$ and $n=4$, the following diagrams depict the equations giving the scale factors (4.9) sending $\omega_{i, i+n}$ to $\tilde{\omega}_{i, i+n}$ for $i$ even. Those for $i$ odd are constructed independently.
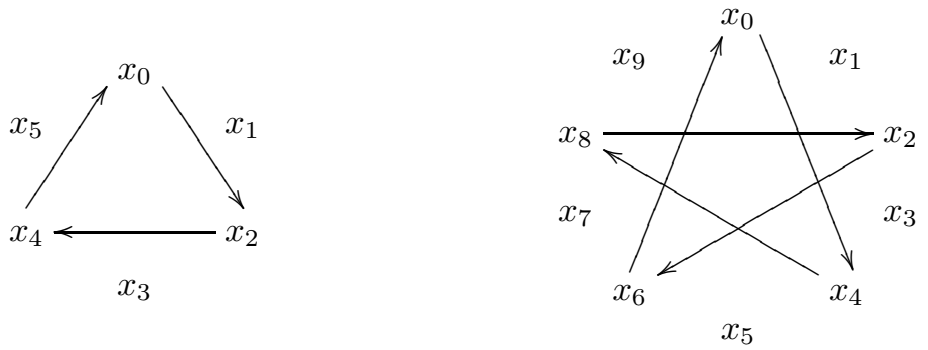

Figure 3. The rescaling scheme for $\mathcal{L}_{2,6}$ and $\mathcal{L}_{4,10}$

Now take $\mathbb{K}=\mathbb{R}$. Because $(2 n+2) / \operatorname{GCD}(n, 2 n+2)=n+1$ is odd, the sign invariant

$$
\operatorname{sgn}\left(\prod_{r=0}^{n} \omega_{r n,(r+1) n}\right)=\operatorname{sgn}\left(\prod_{s=0}^{n} \omega_{2 s, 2 s+n}\right)
$$

reverses under passage to the opposite configuration. Therefore, replacing $\left(\mathbb{R} x_{1}, \ldots, \mathbb{R} x_{2 n+2}\right)$ by its opposite if necessary, we may assume that

$$
\operatorname{sgn}\left(\prod_{s=0}^{n} \omega_{2 s, 2 s+n}\right)=\operatorname{sgn}\left(\prod_{s=0}^{n} \tilde{\omega}_{2 s, 2 s+n}\right) .
$$

Under this assumption we will show that the scale factors (4.9) are all real, so the two configurations are equivalent over $\mathbb{R}$. This will complete the proof of Theorem 1 (ii) for even $n$.

To prove $\lambda_{i}$ real, we must prove $\lambda_{i}^{2}$ positive. From (4.9),

$$
\lambda_{i}^{2}=\prod_{r=0}^{n}\left(\frac{\tilde{\omega}_{i+r n, i+(r+1) n}}{\omega_{i+r n, i+(r+1) n}}\right)^{(-1)^{r}}, \quad \operatorname{sgn}\left(\lambda_{i}^{2}\right)=\operatorname{sgn}\left(\prod_{j \equiv i \bmod 2} \tilde{\omega}_{j, j+n} / \omega_{j, j+n}\right) .
$$

For $i$ even, this is positive by (4.11). To prove it positive for $i$ odd, we must prove that

$$
\operatorname{sgn}\left(\prod_{s=0}^{n} \omega_{2 s+1,2 s+1+n}\right)=\operatorname{sgn}\left(\prod_{s=0}^{n} \tilde{\omega}_{2 s+1,2 s+1+n}\right) .
$$

Check that

$$
\prod_{i=0}^{n} c_{i}=\left(\prod_{i=0}^{n} \omega_{i, i+n+1}\right)^{2}\left(\prod_{i=0}^{2 n+1} \omega_{i, i+n}\right)^{-1} .
$$

Because the two configurations have the same cross-ratios, we must have

$$
\operatorname{sgn}\left(\prod_{i=0}^{2 n+1} \omega_{i, i+n}\right)=\operatorname{sgn}\left(\prod_{i=0}^{2 n+1} \tilde{\omega}_{i, i+n}\right) .
$$


Therefore (4.11) implies (4.12).

The case of $n$ odd. Here $\operatorname{GCD}(n, 2 n+2)=1$, so in contrast with the case that $n$ is even, the entire set of lines in an $(n, 2 n+2)$-configuration may be listed with increments of $n$ :

$$
\left\{\mathbb{K} x_{0}, \mathbb{K} x_{1}, \mathbb{K} x_{2}, \ldots, \mathbb{K} x_{2 n+1}\right\}=\left\{\mathbb{K} x_{0}, \mathbb{K} x_{n}, \mathbb{K} x_{2 n}, \ldots, \mathbb{K} x_{(2 n+1) n}\right\} .
$$

Set $\lambda_{0}:=1$ and define $\lambda_{n}, \ldots, \lambda_{(2 n+1) n}$ recursively by $\lambda_{r n}:=\lambda_{(r-1) n} \tilde{\omega}_{(r-1) n, r n} / \omega_{(r-1) n, r n}$ for $1 \leqslant r \leqslant$ $2 n+1$. This leads to

$$
\lambda_{r n}=\prod_{s=1}^{r}\left(\frac{\tilde{\omega}_{(s-1) n, s n}}{\omega_{(s-1) n, s n}}\right)^{(-1)^{r-s}} .
$$

At this point we have $\lambda_{i} \lambda_{i+n}=\tilde{\omega}_{i, i+n} / \omega_{i, i+n}$ except possibly at $i \equiv-n$ modulo $2 n+2$, where

$$
\lambda_{-n} \lambda_{0}=\prod_{s=1}^{2 n+1}\left(\frac{\tilde{\omega}_{(s-1) n, s n}}{\omega_{(s-1) n, s n}}\right)^{(-1)^{s-1}} .
$$

We claim that this is in fact $\tilde{\omega}_{-n, 0} / \omega_{-n, 0}$. The proof reduces to proving that the expression

$$
\frac{\omega_{0, n} \omega_{2 n, 3 n} \cdots \omega_{2 n^{2},(2 n+1) n}}{\omega_{n, 2 n} \omega_{3 n, 4 n} \cdots \omega_{(2 n+1) n,(2 n+2) n}}=\frac{\omega_{0, n} \omega_{2, n+2} \cdots \omega_{2 n, 3 n}}{\omega_{1, n+1} \omega_{3, n+3} \cdots \omega_{2 n+1,3 n+1}}
$$

does not change if all the $\omega$ 's are replaced with $\tilde{\omega}$ 's. This is true, because as the reader may check, it is equal to

and the $\omega$ 's and $\tilde{\omega}$ 's have the same cross-ratios.

$$
\frac{c_{0} c_{2} \cdots c_{n-1}}{c_{1} c_{3} \cdots c_{n}}
$$

Thus we may replace $x_{i}$ by $\lambda_{i} x_{i}$, giving $\omega_{i, i+n}=\tilde{\omega}_{i, i+n}$ for all $i$. Then by equality of cross-ratios, $\omega_{i, i+n+1} \omega_{i+1, i+n+2}$ is equal to $\tilde{\omega}_{i, i+n+1} \tilde{\omega}_{i+1, i+n+2}$, or, equivalently,

$$
\left(\tilde{\omega}_{i, i+n+1} / \omega_{i, i+n+1}\right)^{(-1)^{i}}
$$

is independent of $i$.

If $\mathbb{K}=\mathbb{C}$, let $\chi$ be a square root of (4.14). If $\mathbb{K}=\mathbb{R}$, let $\chi$ be a square root of its magnitude. Observe that for any $\delta$, the rescaling $x_{i} \mapsto \delta^{(-1)^{i}} x_{i}$ leaves $\omega_{i, i+n}$ unchanged and multiplies $\omega_{i, i+n+1}$ by $\delta^{2(-1)^{i}}$. Therefore in the case $\mathbb{K}=\mathbb{C}$, replacing the $x_{i}$ by $\chi^{(-1)^{i}} x_{i}$ gives $\omega_{i j}=\tilde{\omega}_{i j}$, proving the two configurations equivalent. In the case $\mathbb{K}=\mathbb{R}$, the same argument proves them equivalent when (4.14) is positive.

In the case that $\mathbb{K}=\mathbb{R}$ and (4.14) is negative, this argument leaves us with $\omega_{i, i+n}=\tilde{\omega}_{i, i+n}$ and $\omega_{i, i+n+1}=-\tilde{\omega}_{i, i+n+1}$. Replacing the $x_{i}$ by the additional rescaling $(-1)^{i} x_{i}$ then gives $\omega_{i j}=-\tilde{\omega}_{i j}$, proving the configurations opposite.

4.3. Proof of Theorem 1(iii). Suppose that $c_{0}, \ldots, c_{n}$ are non-zero scalars in $\mathbb{K}$ satisfying (3.2), and extend them $(n+1)$-periodically to $\left(c_{i}\right)_{i \in \mathbb{Z}}$. We will construct an $(n, 2 n+2)$-Lagrangian configuration $\left(\mathbb{K} x_{0}, \mathbb{K} x_{1}, \ldots, \mathbb{K} x_{2 n+1}\right)$ having the given scalars $c_{i}$ as cross-ratios.

As an intermediate step, we construct from the $c_{i}$ scalars $\omega_{i j}$ that will be equal to $\omega\left(x_{i}, x_{j}\right)$. By (2.4) and the Lagrangian condition, it is only necessary to construct a $(2 n+2)$-periodic sequence $\omega_{i, i+n}$ and an $(n+1)$-periodic sequence $\omega_{i, i+n+1}$ such that the $c_{i}$ are given by (2.6): then $\omega_{i, i+n+2}=\omega_{i-n, i}$, and the remaining $\omega_{i j}$ are 0.

The $\omega_{i j}$ for $n$ even. Over $\mathbb{C}$, set $\omega_{i, i+n}:=1$ for all $i$ and fix an $(n+1)$-periodic sequence $\sigma_{i}$ of square roots of the $c_{i}: \sigma_{i}^{2}=c_{i}$. It is then simple to check that (2.6) is satisfied if we set

$$
\omega_{i, i+n+1}:=\frac{\sigma_{i} \sigma_{i+2} \cdots \sigma_{i+n}}{\sigma_{i+1} \sigma_{i+3} \cdots \sigma_{i+n-1}} .
$$

Over $\mathbb{R}$, the same process works if $c_{0} c_{1} \cdots c_{n}$ is positive: the individual $\sigma_{i}$ may not be real, but the $\omega_{i, i+n+1}$ are because their squares are positive. 
If $c_{0} c_{1} \cdots c_{n}$ is negative, it suffices to modify the construction as follows: set $\omega_{i, i+n}:=(-1)^{i}$, let $\sigma_{i}$ be an $(n+1)$-periodic sequence of square roots of the $-c_{i}$, and again define the $\omega_{i, i+n+1}$ by (4.15).

The $\omega_{i j}$ for $n$ odd. Observe that in this case, (2.6) implies $\prod_{i=0}^{2 n+1} \omega_{i, i+n}^{(-1)^{i}}=\prod_{0}^{n} c_{i}^{(-1)^{i}}$.

We begin with an asymmetric choice of the $\omega_{i j}$ that works over any field. Define $\omega_{0, n}:=\prod_{0}^{n} c_{i}^{(-1)^{i}}$, and for $1 \leqslant i \leqslant 2 n+1$, set $\omega_{i, i+n}:=1$. Check that it suffices to set $\omega_{0, n+1}:=1, \omega_{1, n+2}:=c_{0}$, and in general, for $0 \leqslant k \leqslant \frac{1}{2}(n-1)$,

$$
\omega_{2 k, 2 k+n+1}:=\frac{c_{1} c_{3} \cdots c_{2 k-1}}{c_{0} c_{2} \cdots c_{2 k-2}}, \quad \omega_{2 k+1,2 k+n+2}:=\frac{c_{0} c_{2} \cdots c_{2 k}}{c_{1} c_{3} \cdots c_{2 k-1}} .
$$

Over $\mathbb{C}$ it is possible to choose the $\omega_{i j}$ more symmetrically, as described in Section 1.3. Fix an $(n+1)$ periodic sequence $\eta_{i}$ such that $\eta_{i}^{2 n+2}=c_{i}$. Define the $\omega_{i, i+n}$ by

$$
\mu:=\frac{\eta_{0} \eta_{2} \cdots \eta_{n-1}}{\eta_{1} \eta_{3} \cdots \eta_{n}}, \quad \omega_{i, i+n}:=\mu^{(-1)^{i}} .
$$

The reader may check that then (2.6) is satisfied by fixing $\omega_{0, n+1}$ arbitrarily and setting

$$
\omega_{2 k, 2 k+n+1}:=\mu^{4 k} \omega_{0, n+1} \frac{c_{1} c_{3} \cdots c_{2 k-1}}{c_{0} c_{2} \cdots c_{2 k-2}}, \quad \omega_{2 k+1,2 k+n+2}:=\mu^{-4 k-2} \omega_{0, n+1}^{-1} \frac{c_{0} c_{2} \cdots c_{2 k}}{c_{1} c_{3} \cdots c_{2 k-1}}
$$

for $0 \leqslant k \leqslant \frac{1}{2}(n-1)$. Requiring $a_{0} a_{2} \cdots a_{n-1}=a_{1} a_{3} \cdots a_{n}$ leads to the most symmetric choice:

$$
\omega_{i, i+n+1}:=\frac{\eta_{i}^{n} \eta_{i+2}^{n-4} \cdots \eta_{i+n-1}^{-n+2}}{\eta_{i+1}^{n-2} \eta_{i+3}^{n-6} \cdots \eta_{i+n}^{-n}}=\prod_{j=0}^{n} \eta_{i+j}^{(-1)^{j}(n-2 j)} .
$$

The $x_{i}$ for $n$ arbitrary. Suppose now that scalars $\omega_{i j}$ in $\mathbb{K}$ have been chosen so as to satisfy (2.6). From these scalars we will construct an $(n, 2 n+2)$-Lagrangian configuration $\left(\mathbb{K} x_{0}, \ldots, \mathbb{K} x_{2 n+1}\right)$ over $\mathbb{K}$ which satisfies $\omega\left(x_{i}, x_{j}\right)=\omega_{i j}$, and therefore has the given cross-ratios $c_{0}, \ldots, c_{n}$.

The representatives $x_{i}$ are almost standard: we set $x_{i}:=e_{i}$ for $1 \leqslant i \leqslant n$, and

$$
\begin{aligned}
& x_{1+n}:=\omega_{1,1+n} f_{1}, \quad x_{2+n}:=\omega_{1,2+n} f_{1}+\omega_{2,2+n} f_{2}, \\
& x_{i+n}:=\omega_{i-2, i+n} f_{i-2}+\omega_{i-1, i+n} f_{i-1}+\omega_{i, i+n} f_{i}, \quad 3 \leqslant i \leqslant n .
\end{aligned}
$$

It is immediate that with these definitions, $\omega\left(x_{i}, x_{j}\right)=\omega_{i j}$ for $1 \leqslant i, j \leqslant 2 n$.

The requirement that $\omega\left(x_{i}, x_{j}\right)=\omega_{i j}$ for $1 \leqslant i \leqslant 2 n$ and $j=2 n+1$ or $2 n+2$ now determines $x_{2 n+1}$ and $x_{0}=-x_{2 n+2}$. We find that

$$
x_{2 n+1}=\omega_{n-1,2 n+1} f_{n-1}+\omega_{n, 2 n+1} f_{n}+\omega_{1+n, 2 n+1} \sum_{i=1}^{n}(-1)^{i}\left(\prod_{j=1}^{i} \omega_{j, j+n}^{-1}\right) d_{i} e_{i}
$$

where $d_{1}=1, d_{2}=\omega_{1,2+n}$, and the $d_{i}$ with $3 \leqslant i \leqslant n$ satisfy the recursion relation

$$
d_{i}=\omega_{i-1, i+n} d_{i-1}-\omega_{i-1, i+n-1} \omega_{i-2, i+n} d_{i-2} .
$$

In the same way we obtain

$$
x_{0}=-\omega_{0, n} f_{n}-\sum_{i=1}^{n}(-1)^{i}\left(\prod_{j=1}^{i} \omega_{j, j+n}^{-1}\right) d_{i}^{\prime} e_{i}
$$

where $d_{1}^{\prime}=\omega_{0,1+n}, d_{2}^{\prime}=\omega_{0,1+n} \omega_{1,2+n}-\omega_{0,2+n} \omega_{1,1+n}$, and the $d_{i}^{\prime}$ with $3 \leqslant i \leqslant n$ also satisfy (4.16). 
In order to clarify (4.16), consider for any integers $0 \leqslant j \leqslant i \leqslant n$ the following truncation of the tridiagonal matrix $A$ in (4.4):

$$
A_{j, i}=\left(\begin{array}{ccccc}
\omega_{j, j+n+1} & \omega_{j, j+n+2} & & & \\
\omega_{j+1, j+n+1} & \omega_{j+1, j+n+2} & \omega_{j+1, j+n+3} & & \\
& \ddots & \ddots & \ddots & \\
& & \omega_{i-1, i+n-1} & \omega_{i-1, i+n} & \omega_{i-1, i+n+1} \\
& & & \omega_{i, i+n} & \omega_{i, i+n+1}
\end{array}\right) .
$$

Let us write $\Delta_{j, i}$ for $\operatorname{det}\left(A_{j, i}\right)$, and adopt the convention $\Delta_{j, j-1}:=1$ and $\Delta_{j, j-2}:=0$. It is clear that the $\Delta_{j, i}$ satisfy a shifted version of (4.16):

$$
\Delta_{j, i}=\omega_{i, i+n+1} \Delta_{j, i-1}-\omega_{i, i+n} \omega_{i-1, i+n+1} \Delta_{j, i-2} .
$$

Therefore $d_{i}=\Delta_{1, i-1}$ and $d_{i}^{\prime}=\Delta_{0, i-1}$ for $1 \leqslant i \leqslant n$.

The only remaining condition is $\omega\left(x_{0}, x_{2 n+1}\right)=0$. After some simplification it reduces to

$$
\Delta_{0, n}-\omega_{0, n} \omega_{n+1,2 n+1} \Delta_{1, n-1}=0 .
$$

Recall the matrices $\Omega_{n}$ and $A$ from (4.3). Because $A$ is $A_{0, n}$, Proposition 4.2 and (4.7) show that (4.17) is equivalent to (4.8). Because we assumed that the given $c_{i}$ satisfy (3.2), these conditions hold. This completes the proof of Theorem 1 .

\section{Normalized COnfigurations}

As noted in Section 3.3, in this section we describe certain normalized choices of representatives of $(n, 2 n+2)$-configurations. As in that section, let $\left(X_{0}, \ldots, X_{2 n+1}\right)$ be a generic $(n, 2 n+2)$-Lagrangian configuration over $\mathbb{K}$ with representatives $x_{0}, \ldots, x_{2 n+1}$, symplectic products $\omega_{i j}:=\omega\left(x_{i}, x_{j}\right)$, and crossratios $c_{0}, \ldots, c_{n}$.

5.1. The case of $n$ even and $\mathbb{K}=\mathbb{C}$. The results in this case were stated in Section 3.3 Here we give their proofs.

Proof of Proposition 3.3. By the constructions in Section 4.3 there exists a generic configuration $\left(\mathbb{C} \tilde{x}_{0}, \ldots, \mathbb{C} \tilde{x}_{2 n+1}\right)$ with $\tilde{\omega}_{i, i+n}=1$ and cross-ratios $c_{0}, \ldots, c_{n}$. By Theorem 1(ii), it is equivalent to $\left(X_{0}, \ldots, X_{2 n+1}\right)$. The images $x_{0}, \ldots, x_{2 n+1}$ of $\tilde{x}_{0}, \ldots, \tilde{x}_{2 n+1}$ under the equivalence have $\omega_{i, i+n}=1$ for all $i$.

To see that there are exactly four such choices of $\left(x_{i}\right)_{i}$, suppose that $\left(\lambda_{i} x_{i}\right)_{i}$ is another. Observe that then $\lambda_{i+n}=\lambda_{i}^{-1}$, so $\lambda_{i+r n}=\lambda_{i}^{(-1)^{r}}$. But $\lambda_{i+n(n+1)}=\lambda_{i}$ by periodicity, so $\lambda_{i}= \pm 1$, whence $\lambda_{i+n}=\lambda_{i}$, and $\lambda_{i}=\lambda_{j}$ for $i \equiv j$ modulo 2 . This proves (i). The remaining statements are immediate.

Proof of Theorem 2, Part (i) follows from Section 4.1 by Lemma 4.3 for normalized representatives $\left(x_{0}, \ldots, x_{2 n+1}\right)$ the Pfaffian of the $\omega$-Gram matrix is, up to a sign, $R_{n+1}\left(a_{0}, \ldots, a_{n}\right)$. Parts (ii) and (iii) follow from Theorem 1(ii) and (iii) and Proposition 3.3(iii): the cross-ratios determine $\pm\left(a_{0}, \ldots, a_{n}\right)$ and vice versa.

5.2. The case of $n$ even and $\mathbb{K}=\mathbb{R}$. Recall from Section 4.2 the sign invariants

$$
\varepsilon_{0}:=\operatorname{sgn}\left(\prod_{s=0}^{n} \omega_{2 s, 2 s+n}\right), \quad \varepsilon_{1}:=\operatorname{sgn}\left(\prod_{s=0}^{n} \omega_{2 s+1,2 s+1+n}\right), \quad \varepsilon_{c}:=\operatorname{sgn}\left(\prod_{i=0}^{n} c_{i}\right)
$$

of the configuration $\left(X_{0}, \ldots, X_{2 n+1}\right): \varepsilon_{0}$ is (4.10), $\varepsilon_{1}$ is the left side of (4.12), and by (4.13), $\varepsilon_{c}=\varepsilon_{0} \varepsilon_{1}$.

Proposition 5.1. For $n$ even and $\mathbb{K}=\mathbb{R},\left(X_{0}, \ldots, X_{2 n+1}\right)$ admits exactly four choices of representatives whose symplectic subdiameters are $\omega_{i, i+n}=\varepsilon_{i \bmod 2}$. Fix such a choice, $x_{0}, \ldots, x_{2 n+1}$, and denote its symplectic diameters $\omega_{i, i+n+1}$ by $a_{i}^{\mathbb{R}}$. 
(i) In terms of $\left(x_{i}\right)_{i}$, the four choices are as in Proposition 3.3(i). The first two have symplectic diameters $\left(a_{i}^{\mathbb{R}}\right)_{i}$, and the second two have symplectic diameters $\left(-a_{i}^{\mathbb{R}}\right)_{i}$. In particular, $\pm\left(a_{i}^{\mathbb{R}}\right)_{i}$ is an invariant of the configuration, the collection of its normalized real symplectic diameters.

(ii) If $\varepsilon_{c}=1$, then the normalized symplectic diameters coincide with the normalized real symplectic diameters: $\pm\left(a_{i}\right)_{i}= \pm\left(a_{i}^{\mathbb{R}}\right)_{i}$.

(iii) If $\varepsilon_{c}=-1$, then $\pm\left(a_{i}\right)_{i}= \pm \sqrt{-1}\left(a_{i}^{\mathbb{R}}\right)_{i}$.

Proof. By the constructions in Section 4.3 , there exists a generic configuration $\left(\mathbb{R} \tilde{x}_{0}, \ldots, \mathbb{R} \tilde{x}_{2 n+1}\right)$ with cross-ratios $c_{i}$, such that if $\varepsilon_{c}=1$, then $\tilde{\omega}_{i, i+n}=1$ for all $i$, and if $\varepsilon_{c}=-1$, then $\tilde{\omega}_{i, i+n}=(-1)^{i}$ for all $i$. By Theorem 1(ii), this configuration is equivalent either to $\left(X_{0}, \ldots, X_{2 n+1}\right)$ or its opposite, and so, replacing $\left(\mathbb{R} \tilde{x}_{0}, \ldots, \mathbb{R} \tilde{x}_{2 n+1}\right)$ by its opposite if necessary, we may assume that it is equivalent to $\left(X_{0}, \ldots, X_{2 n+1}\right)$. Then, recalling that passage to opposites negates symplectic products, we find that the images $x_{0}, \ldots, x_{2 n+1}$ of $\tilde{x}_{0}, \ldots, \tilde{x}_{2 n+1}$ under the equivalence have $\omega_{i, i+n}=\varepsilon_{i \bmod 2}$.

The proof of (i) goes exactly as in Proposition 3.3. For (ii) and (iii), fix a choice of $\sqrt{-1}$. In (ii), if $\varepsilon_{0}$ and $\varepsilon_{1}$ are both -1 , then the representatives of the complex normalization of Section 3.3 may be taken to be $\sqrt{-1}\left(x_{i}\right)_{i}$, while if they are both 1 , then the real and complex normalizations coincide.

In (iii), if $\varepsilon_{0}=1$ and $\varepsilon_{1}=-1$, the $\mathbb{C}$-normalized representatives may be taken to be $x_{i}$ for $i$ even and $\sqrt{-1} x_{i}$ for $i$ odd, while if $\varepsilon_{0}=-1$ and $\varepsilon_{1}=1$, they may be taken to be $\sqrt{-1} x_{i}$ for $i$ even and $x_{i}$ for $i$ odd. To summarize, in all cases the $\mathbb{C}$-normalized representatives are $\left(\sqrt{-1}^{\left(1-\varepsilon_{i \bmod 2}\right) / 2} x_{i}\right)_{i}$. The relation between $\pm\left(a_{i}\right)_{i}$ and $\pm\left(a_{i}^{\mathbb{R}}\right)_{i}$ now follows easily.

5.3. The case of $n$ odd and $\mathbb{K}=\mathbb{C}$. Recall from Section 4.3 that here $\prod_{i=0}^{2 n+1} \omega_{i, i+n}^{(-1)^{i}}=\prod_{0}^{n} c_{i}^{(-1)^{i}}$.

Proposition 5.2. For $n$ odd, $\mathbb{K}=\mathbb{C}$, and $\mu$ any $(2 n+2)^{\text {nd }}$ root of $\prod_{0}^{n} c_{i}^{(-1)^{i}},\left(X_{0}, \ldots, X_{2 n+1}\right)$ admits exactly $(2 n+2)$ choices of representatives such that $\omega_{i, i+n}=\mu^{(-1)^{i}}$ for all $i$ and $\prod_{0}^{n} \omega_{i, i+n+1}^{(-1)^{i}}=1$. Fix such a choice, $x_{0}, \ldots, x_{2 n+1}$, and set $a_{i}:=\omega_{i, i+n+1}$.

(i) The $2 n+2$ choices are $\left(\delta^{(-1)^{i}} x_{i}\right)_{i}$, where $\delta$ runs over the $(2 n+2)^{\text {nd }}$ roots of unity.

(ii) The symplectic diameters corresponding to any given choice of $\delta$ are $\left(\delta^{2(-1)^{i}} a_{i}\right)_{i}$.

(iii) The cross-ratios of the configuration are $c_{i}=\mu^{2(-1)^{i}} a_{i} a_{i+1}$.

Proof. The discussion in Section 4.3 shows that for any $(2 n+2)^{\text {nd }}$ root $\mu$ of $\prod_{0}^{n} c_{i}^{(-1)^{i}}$, there exists a configuration $\left(\mathbb{C} \tilde{x}_{0}, \ldots, \mathbb{C} \tilde{x}_{2 n+1}\right)$ with $\tilde{\omega}_{i, i+n}=\mu^{(-1)^{i}}$ and cross-ratios $c_{0}, \ldots, c_{n}$. By Theorem 1 (ii), it is equivalent to $\left(X_{0}, \ldots, X_{2 n+1}\right)$. The images $x_{0}, \ldots, x_{2 n+1}$ of $\tilde{x}_{0}, \ldots, \tilde{x}_{2 n+1}$ under the equivalence have $\omega_{i, i+n}=\mu^{(-1)^{i}}$ for all $i$.

For (i), first check that if a renormalization $x_{i} \mapsto \lambda_{i} x_{i}$ preserves $\omega_{i, i+n}$ for all $i$, then it is of the form $\lambda_{i}=\delta^{(-1)^{i}}$ for some $\delta$. Then check that $\prod_{0}^{n} \omega_{i, i+n+1}^{(-1)^{i}}=1$ if and only if $\delta^{2 n+2}=1$. The remaining statements are clear.

We remark that the general rescaling $x_{i} \mapsto \lambda_{i} x_{i}$ going between normalizations as above with different choices of $\mu$ is $\lambda_{i}:=\xi^{(-1)^{i}(i p+q)}$, where $\xi$ is any primitive $(2 n+2)^{\text {nd }}$ root of unity and $p$ and $q$ are arbitrary elements of $\mathbb{Z}_{2 n+2}$. It transforms $\omega_{i, i+n}$ from $\mu^{(-1)^{i}}$ to $\left(\xi^{-p n} \mu\right)^{(-1)^{i}}$ and $\omega_{i, i+n+1}$ from $a_{i}$ to $(-1)^{p} \xi^{2(-1)^{i}(i p+q)} a_{i}$, i.e.,

$$
\left(\mu, a_{i}\right)_{i} \mapsto\left(\xi^{-p n} \mu,(-1)^{p} \xi^{2(-1)^{i}(i p+q)} a_{i}\right)_{i} .
$$

We will not formally state the specialization of Theorem 1 corresponding to the normalization in Proposition [5.2. but let us describe the specialization of the relation (3.2): it becomes the vanishing of the quantity obtained from the product $a_{0} \cdots a_{n}$ by applying the " $\mu$-cyclic replacement rule": replace 
cyclically adjacent pairs $a_{i} a_{i+1}$ by $-\mu^{-2(-1)^{i}}$. For example, at $n=1$ and 3 ,

$$
\begin{aligned}
& 0=a_{0} a_{1}-\mu^{-2}-\mu^{2}, \\
& 0=a_{0} a_{1} a_{2} a_{3}-\mu^{-2}\left(a_{0} a_{1}+a_{2} a_{3}\right)+\mu^{-4}+\mu^{4} .
\end{aligned}
$$

5.4. The case of $n$ odd and $\mathbb{K}=\mathbb{R}$. Here we have only found natural normalizations under certain positivity conditions.

Proposition 5.3. Suppose that $n$ is odd, $\mathbb{K}=\mathbb{R}$, and $\prod_{0}^{n} c_{i}^{(-1)^{i}}$ is positive, and let $\mu$ be its positive $(2 n+2)^{\text {nd }}$ root. Then $\left(X_{0}, \ldots, X_{2 n+1}\right)$ admits choices of representatives such that $\omega_{i, i+n}=\mu^{(-1)^{i}}$ for all $i$.

If both $c_{0} c_{2} \cdots c_{n+1}$ and $c_{1} c_{3} \cdots c_{n}$ are positive, then exactly two such choices satisfy in addition $\prod_{0}^{n} \omega_{i, i+n+1}^{(-1)^{i}}=1$. Otherwise there is no such choice.

Proof. Given any representatives $\left(x_{i}\right)_{i}$, rescale to $\lambda_{i} x_{i}$, where $\lambda_{0}:=1$ and

$$
\lambda_{r n} \lambda_{(r+1) n} \omega_{r n,(r+1) n}=\mu^{(-1)^{i}}
$$

for $0<r<2 n+2$. Check that this proves the first paragraph.

In the second paragraph we can only use further rescalings preserving the subdiameters: $x_{i} \mapsto \delta^{(-1)^{i}} x_{i}$. Such rescalings multiply $\prod_{0}^{n} \omega_{i, i+n+1}^{(-1)^{i}}$ by $\delta^{2 n+2}$, so we can choose $\delta$ to make the product 1 if and only if $\prod_{0}^{n} \omega_{i, i+n+1}$ is positive. To complete the proof, observe that $c_{0} c_{2} \cdots c_{n-1}$ is $\mu^{n+1} \prod_{0}^{n} \omega_{i, i+n+1}$. There are two choices because the sign of $\delta$ is irrelevant.

\section{Symmetric linear difference equations and the Closure of $\mathcal{L}_{n, N}(\mathbb{K})$}

In this section we present general results relating Lagrangian configurations to non-degenerate symmetric linear difference equations of degree $2 n$. The solution space of such an equation has a natural symplectic form, generalizing the Wronski determinant. When the equation has $N$-periodic coefficients and monodromy - Id, there is a simple way to construct a particular Lagrangian configuration in its solution space. This yields a projection from the space of all such equations to equivalence classes of Lagrangian configurations.

6.1. Linear difference operators. Let $T$ be the shift operator, acting on infinite sequences $\left(V_{i}\right)_{i \in \mathbb{Z}}$ by $(T V)_{i}:=V_{i-1}$. A linear difference operator over $\mathbb{K}$ is a polynomial expression in $T$ and its inverse,

$$
A=a^{n} T^{n}+a^{n-1} T^{n-1}+\cdots+a^{m} T^{m},
$$

where $m \leqslant n$ are arbitrary integers and the coefficients $a^{\ell}$ are sequences $\left(a_{i}^{\ell}\right)_{i \in \mathbb{Z}}$ of $\mathbb{K}$-scalars. Such operators act on sequences $\left(V_{i}\right)$ of $\mathbb{K}$-scalars, the coefficients acting by multiplication: $(a V)_{i}:=a_{i} V_{i}$.

- $A$ is said to be of order $n-m$ if both $a^{m}$ and $a^{n}$ are non-zero.

- $A$ is said to be non-degenerate if both $a_{i}^{m}$ and $a_{i}^{n}$ are non-zero for all $i$.

- $A$ is said to be $N$-periodic if $a_{i}^{\ell}=a_{i+N}^{\ell}$ for all $\ell$ and $i$.

Definition. The adjoint $A^{*}$ of a linear difference operator $A$ is defined by

$$
V \cdot\left(A^{*} W\right)=(A V) \cdot W,
$$

where $V \cdot W:=\sum_{i \in \mathbb{Z}} V_{i} W_{i}$, an inner product on scalar sequences with only finitely many non-zero terms.

It is simple to check that $T^{*}=T^{-1}$. This is the discrete analog of the fact that translation is the exponential of the derivation $\frac{d}{d x}$, and $\frac{d}{d x}^{*}=-\frac{d}{d x}$. It is also clear that $(A B)^{*}=B^{*} A^{*}$ for any operators $A$ and $B$. In particular, writing $\left(T^{\ell^{\prime}} a^{\ell}\right)$ for the multiplication operator $\left(T^{\ell^{\prime}} a^{\ell}\right)_{i}=a_{i-\ell^{\prime}}^{\ell}$, one obtains the following lemma.

Lemma 6.1. $\left(\sum_{\ell=m}^{n} a^{\ell} T^{\ell}\right)^{*}=\sum_{\ell=m}^{n}\left(T^{-\ell} a^{\ell}\right) T^{-\ell}$. 
Definition. If an operator $A$ satisfies $A=A^{*}$, it is self-adjoint, or symmetric. In this case, for some $n \geqslant 0$ there exist sequences $a^{0}, \ldots, a^{n}$ such that

$$
A=a^{0}+\sum_{\ell=1}^{n}\left(a^{\ell} T^{\ell}+\left(T^{-\ell} a^{\ell}\right) T^{-\ell}\right) .
$$

Remark. The spectral theory of linear difference operators is quite similar to that of linear differential operators; see [10] and references therein. Operators with periodic or antiperiodic solutions play a special role in [10], where they are called "superperiodic".

6.2. Linear difference equations. The linear difference equation corresponding to a linear difference operator $A$ is $A V=0$. We denote the space of solutions of this equation, the kernel of $A$, by $\mathcal{K}(A)$ :

$$
\mathcal{K}(A):=\{V: A V=0\} .
$$

Lemma 6.2. Let $A$ be a non-degenerate linear difference operator over $\mathbb{K}$ of order $p$. For any $i_{0} \in \mathbb{Z}$ and any $\mathbb{K}$-scalars $c_{i_{0}+1}, \cdots, c_{i_{0}+p}$, there is a unique solution $\left(V_{i}\right)$ of the equation $A V=0$ satisfying the initial conditions $V_{i}=c_{i}$ for $i_{0}<i \leqslant i_{0}+p$. In particular, $\mathcal{K}(A)$ is a $p$-dimensional vector space over $\mathbb{K}$.

The proof of this lemma is immediate. Note that the symmetric operator (6.2) is non-degenerate if and only if $a_{i}^{n} \neq 0$ for all $i$. Let us write the equation $A V=0$ explicitly in this case:

$$
a_{i}^{n} V_{i-n}+\cdots+a_{i}^{1} V_{i-1}+a_{i}^{0} V_{i}+a_{i+1}^{1} V_{i+1}+\cdots+a_{i+n}^{n} V_{i+n}=0 \text { for all } i .
$$

Corollary 6.3. Given a non-degenerate symmetric difference operator $A$ over $\mathbb{K}$ of degree $2 n$ as in (6.3), for all $i \in \mathbb{Z}$ there is a unique element $V^{i}(A)$ of the kernel $\mathcal{K}(A)$ such that

$$
\left(V_{i-n}^{i}(A), V_{i-n+1}^{i}(A), \ldots, V_{i+n-1}^{i}(A), V_{i+n}^{i}(A)\right):=\left(-\frac{1}{a_{i}^{n}}, 0,0, \ldots, 0, \frac{1}{a_{i+n}^{n}}\right) .
$$

For any $i,\left\{V^{i+1}(A), V^{i+2}(A), \ldots, V^{i+2 n}(A)\right\}$ is a basis of $\mathcal{K}(A)$.

An important property of non-degenerate symmetric operators is the existence of a natural symplectic form on their kernels. Before giving the general result, we describe the simplest case.

Example. The operator $L:=T-a+T^{-1}$ is known as the discrete Sturm-Liouville (or Hill, or Schrödinger) operator. It is non-degenerate and symmetric, and the classical Wronski determinant

$$
\mathcal{W}\left(V, V^{\prime}\right):=\left|\begin{array}{cc}
V_{i-1} & V_{i-1}^{\prime} \\
V_{i} & V_{i}^{\prime}
\end{array}\right|
$$

is a well-defined symplectic form on its kernel $\mathcal{K}(L)$. To understand this, check that when $L(V)$ and $L\left(V^{\prime}\right)$ are zero, $\mathcal{W}\left(V, V^{\prime}\right)$ is independent of the choice of $i$ :

$$
\left|\begin{array}{cc}
V_{i} & V_{i}^{\prime} \\
V_{i+1} & V_{i+1}^{\prime}
\end{array}\right|=-\left|\begin{array}{cc}
V_{i} & V_{i}^{\prime} \\
V_{i-1} & V_{i-1}^{\prime}
\end{array}\right|-a_{i}\left|\begin{array}{cc}
V_{i} & V_{i}^{\prime} \\
V_{i} & V_{i}^{\prime}
\end{array}\right|=\left|\begin{array}{cc}
V_{i-1} & V_{i-1}^{\prime} \\
V_{i} & V_{i}^{\prime}
\end{array}\right| .
$$

Remark. The continuant (3.3) may be viewed as an element of $\mathcal{K}(L)$ : the Sturm-Liouville difference equation is

$$
V_{i-1}-a_{i} V_{i}+V_{i+1}=0,
$$

and the initial conditions $\left(V_{-1}, V_{0}\right)=(0,1)$ give $V_{n}=K_{n}\left(a_{0}, \ldots, a_{n-1}\right)$. In fact, continuants are the simplest members of the series of André determinants, which satisfy linear difference equations of higher order; see [1] and also [13].

We now define a multidimensional version of the Wronski determinant. It is a discrete analog of the symplectic form on the solution space of the symmetric linear differential equation studied in [16]. 
Definition. Fix a non-degenerate symmetric linear difference operator $A$ over $\mathbb{K}$ of order $2 n$, as in (6.2). Given two elements $V$ and $V^{\prime}$ of the $\operatorname{kernel} \mathcal{K}(A)$ and any $i \in \mathbb{Z}$, set

$$
\mathcal{W}_{A}^{i}\left(V, V^{\prime}\right):=\sum_{\ell=1}^{n} \sum_{m=i+1}^{i+\ell} a_{m}^{\ell}\left|\begin{array}{cc}
V_{m-\ell} & V_{m-\ell}^{\prime} \\
V_{m} & V_{m}^{\prime}
\end{array}\right| .
$$

Lemma 6.4. (i) $\mathcal{W}_{A}^{i}$ is independent of $i$ and is a symplectic form $\mathcal{W}_{A}$ on $\mathcal{K}(A)$.

(ii) Writing $V^{i}$ for the solution $V^{i}(A)$ of Corollary [6.3, $\mathcal{W}_{A}\left(V^{i}, V^{j}\right)=V_{j}^{i}=-V_{i}^{j}$.

(iii) In particular, $\mathcal{W}_{A}\left(V^{i}, V^{j}\right)=0$ for $|i-j|<n$, and $\mathcal{W}_{A}\left(V^{j-n}, V^{j}\right)=1 / a_{j}^{n}$.

Proof. Let us use the shorthand $\left|\begin{array}{l}j \\ k\end{array}\right|$ for $\left|\begin{array}{ll}V_{j} & V_{j}^{\prime} \\ V_{k} & V_{k}^{\prime}\end{array}\right|$. It is helpful to expand $\mathcal{W}_{A}^{i}\left(V, V^{\prime}\right)$ as

$$
\begin{aligned}
& \left(a_{i+1}^{1}\left|\begin{array}{c}
i \\
i+1
\end{array}\right|\right)+\left(a_{i+1}^{2}\left|\begin{array}{c}
i-1 \\
i+1
\end{array}\right|+a_{i+2}^{2}\left|\begin{array}{c}
i \\
i+2
\end{array}\right|\right)+\left(a_{i+1}^{3}\left|\begin{array}{c}
i-2 \\
i+1
\end{array}\right|+a_{i+2}^{3}\left|\begin{array}{c}
i-1 \\
i+2
\end{array}\right|+a_{i+3}^{3}\left|\begin{array}{c}
i \\
i+3
\end{array}\right|\right) \\
& +\cdots+\left(a_{i+1}^{n}\left|\begin{array}{c}
i+1-n \\
i+1
\end{array}\right|+a_{i+2}^{n}\left|\begin{array}{c}
i+2-n \\
i+2
\end{array}\right|+\cdots+a_{i+n}^{n}\left|\begin{array}{c}
i \\
i+n
\end{array}\right|\right) .
\end{aligned}
$$

To prove that $\mathcal{W}_{A}^{i}$ is independent of $i$, verify that $\mathcal{W}_{A}^{i}\left(V, V^{\prime}\right)-\mathcal{W}_{A}^{i-1}\left(V, V^{\prime}\right)$ is

$$
\left(a_{i+1}^{1}\left|\begin{array}{c}
i \\
i+1
\end{array}\right|-a_{i}^{1}\left|\begin{array}{c}
i-1 \\
i
\end{array}\right|\right)+\left(a_{i+2}^{2}\left|\begin{array}{c}
i \\
i+2
\end{array}\right|-a_{i}^{2}\left|\begin{array}{c}
i-2 \\
i
\end{array}\right|\right)+\cdots+\left(a_{i+n}^{n}\left|\begin{array}{c}
i \\
i+n
\end{array}\right|-a_{i}^{n}\left|\begin{array}{c}
i-n \\
i
\end{array}\right|\right) .
$$

Convert $\left|\begin{array}{c}i-\ell \\ i\end{array}\right|$ to $-\left|\begin{array}{c}i \\ i-\ell\end{array}\right|$ and use (6.3) $),\left|\begin{array}{l}i \\ i\end{array}\right|=0$, and $A V=A V^{\prime}=0$ to check that this is

$$
\left|\begin{array}{cc}
V_{i} & V_{i}^{\prime} \\
(A V)_{i} & \left(A V^{\prime}\right)_{i}
\end{array}\right|=\left|\begin{array}{cc}
V_{i} & V_{i}^{\prime} \\
0 & 0
\end{array}\right|=0
$$

Thus we may write simply $\mathcal{W}_{A}$ for $\mathcal{W}_{A}^{i}$. Clearly it is a skew-symmetric bilinear form on $\mathcal{K}(A)$.

For (ii) and (iii), it suffices to check from the definitions that for any $V^{\prime} \in \mathcal{K}(A)$,

$$
\mathcal{W}_{A}^{i-1}\left(V^{i}, V^{\prime}\right)=a_{i}^{n}\left|\begin{array}{cc}
V_{i-n}^{i} & V_{i-n}^{\prime} \\
V_{i}^{i} & V_{i}^{\prime}
\end{array}\right|=-V_{i}^{\prime}
$$

To prove that $\mathcal{W}_{A}$ is non-degenerate, recall Lemma 2.1 and consider the matrix $\Omega_{\mathcal{W}_{A}}$ of $\mathcal{W}_{A}$ in the basis $\left\{V^{j-n+1}, V^{j+1}, \ldots, V^{j+n}\right\}$ : for $1 \leqslant r, s \leqslant 2 n,\left(\Omega_{\mathcal{W}_{A}}\right)_{r s}:=\mathcal{W}_{A}\left(V^{r+j-n}, V^{s+j-n}\right)$. The result will follow if we prove $\operatorname{det}\left(\Omega_{\mathcal{W}_{A}}\right) \neq 0$. Applying (iii), we find

$$
\Omega_{\mathcal{W}_{A}}=\left(\begin{array}{cc}
0 & T \\
-T^{t} & 0
\end{array}\right)
$$

where $T$ is an $n \times n$ upper triangular matrix with diagonal entries $\left(a_{j+1}^{n}\right)^{-1},\left(a_{j+2}^{n}\right)^{-1}, \ldots,\left(a_{j+n}^{n}\right)^{-1}$.

Rescaling. Suppose that $\lambda$ is a non-vanishing sequence over $\mathbb{K}$ : a sequence $\left(\lambda_{i}\right)_{i \in \mathbb{Z}}$ of non-zero $\mathbb{K}$-scalars. Given an operator $A$, we define its rescaling by $\lambda$ to be the operator $\lambda^{-1} \circ A \circ \lambda^{-1}$.

Lemma 6.5. Let $A$ be a non-degenerate symmetric linear difference operator over $\mathbb{K}$ of order $2 n$, as in (6.2), and let $\lambda$ be a non-vanishing sequence over $\mathbb{K}$. Let $\tilde{A}$ be the rescaling $\lambda^{-1} A \lambda^{-1}$.

(i) $\tilde{A}$ is a non-degenerate symmetric operator over $\mathbb{K}$ of order $2 n$. Its coefficients $\tilde{a}^{\ell}$ are

$$
\tilde{a}_{i}^{\ell}=\lambda_{i}^{-1} \lambda_{i-\ell}^{-1} a_{i}^{\ell} .
$$

(ii) If $A$ and $\lambda$ are $N$-periodic, then $\tilde{A}$ is too.

(iii) $\lambda$ is a symplectic map from $\left(\mathcal{K}(A), \mathcal{W}_{A}\right)$ to $\left(\mathcal{K}(\tilde{A}), \mathcal{W}_{\tilde{A}}\right)$.

(iv) $\lambda\left(V^{i}(A)\right)=\lambda_{i}^{-1} V^{i}(\tilde{A})$. 
Proof. We leave (i), (ii), and $\lambda(\mathcal{K}(A))=\mathcal{K}(\tilde{A})$ to the reader. To prove $\lambda$ symplectic, verify

$$
\mathcal{W}_{\tilde{A}}^{i}\left(\lambda V, \lambda V^{\prime}\right):=\sum_{\ell=1}^{n} \sum_{m=i+1}^{i+\ell} \tilde{a}_{m}^{\ell} \lambda_{m} \lambda_{m-\ell}\left|\begin{array}{cc}
V_{m-\ell} & V_{m-\ell}^{\prime} \\
V_{m} & V_{m}^{\prime}
\end{array}\right| .
$$

Because $\tilde{a}_{m}^{\ell} \lambda_{m} \lambda_{m-\ell}=a_{m}^{\ell}$, this is simply $\mathcal{W}_{A}^{i}\left(V, V^{\prime}\right)$.

For (iv), use Corollary 6.3 to check that $\lambda\left(V^{i}(A)\right)_{j}=\lambda_{i}^{-1} V^{i}(\tilde{A})_{j}$ for $i-n \leqslant j \leqslant i+n$. By (iii), both $\lambda\left(V^{i}(A)\right)$ and $\lambda_{i}^{-1} V^{i}(\tilde{A})$ are in $\mathcal{K}(\tilde{A})$, so by Lemma 6.2 they are equal.

6.3. Periodic operators, monodromy, and Lagrangian configurations. Difference equations corresponding to $N$-periodic operators do not necessarily have $N$-periodic solutions. However, we do have the following lemma. Its proof is immediate from the obvious fact that an operator is $N$-periodic if and only if it commutes with $T^{N}$.

Lemma 6.6. Suppose that $A$ is an $N$-periodic linear difference operator. Then $T^{N}$ preserves the kernel $\mathcal{K}(A)$. It is called the monodromy operator $M_{A}$ of $A$ :

$$
M_{A}:=\left.T^{N}\right|_{\mathcal{K}(A)}: \mathcal{K}(A) \rightarrow \mathcal{K}(A) .
$$

In the case of non-degenerate symmetric operators, the monodromy is symplectic:

Lemma 6.7. Suppose that $A$ is a non-degenerate $N$-periodic symmetric linear difference operator of order $2 n$. Then the monodromy operator $M_{A}$ preserves the symplectic form $\mathcal{W}_{A}$ on $\mathcal{K}(A)$.

Proof. We must prove that $\mathcal{W}_{A}\left(T^{N} V, T^{N} V^{\prime}\right)=\mathcal{W}_{A}\left(V, V^{\prime}\right)$ for all elements $V$ and $V^{\prime}$ of $\mathcal{K}(A)$. Recall that $\mathcal{W}_{A}$ may be expressed as $\mathcal{W}_{A}^{i}$ for any $i$. Use the fact that $\left(T^{N} a^{\ell}\right)=a^{\ell}$ for all $\ell$ to check that $\mathcal{W}_{A}^{i}\left(T^{N} V, T^{N} V^{\prime}\right)=\mathcal{W}_{A}^{i-N}\left(V, V^{\prime}\right)$.

Our main result in Section 6 is Theorem 3 , the most general result of the paper. It states that a certain set of difference operators may be projected to symplectic equivalence classes of Lagrangian configurations. In order to define this projection we make two preliminary definitions.

Definition. For $N \geqslant 2 n$, let $\overline{\mathcal{L}}_{n, N}(\mathbb{K})$ be the $\operatorname{Sp}(2 n, \mathbb{K})$-moduli space of symplectic equivalence classes of all $(n, N)$-Lagrangian configurations over $\mathbb{K}$, both generic and non-generic.

Definition. For $N \geqslant 2 n$, let $\mathcal{E}_{n, N}(\mathbb{K})$ be the set of non-degenerate $N$-periodic symmetric linear difference operators over $\mathbb{K}$ of order $2 n$ with monodromy -Id.

Remarks. - Regarded as a subset of $\left(\mathbb{K} \mathbb{P}^{2 n-1}\right)^{N}, \mathcal{L}_{n, N}(\mathbb{K})$ is dense in $\overline{\mathcal{L}}_{n, N}(\mathbb{K})$ in both the standard and Zariski topologies.

- For both geometric and analytic reasons, imposing the condition that the monodromy be Id in the definition of $\mathcal{E}_{n, N}(\mathbb{K})$ would be less natural; cf. [13, 10, for the SL(2n)-analog.

- Suppose that $A$ is a non-degenerate $N$-periodic symmetric linear difference operator of order $2 n$. Fix initial conditions $V_{i_{0}+1}, \ldots, V_{i_{0}+2 n}$, and let $V$ be the corresponding solution of $A V=0$. It is easy to see that each entry $V_{i}$ of $V$ depends polynomially on the quantities $\left(a_{k}^{n}\right)^{ \pm 1}, a_{k}^{n-1}, \ldots, a_{k}^{0}$ for $i_{0}<k \leqslant i_{0}+N$. It follows that the same is true of $M_{A}$, and so $\mathcal{E}_{n, N}$ is an algebraic variety.

We will see that $\mathcal{E}_{n, N}$ projects to $\overline{\mathcal{L}}_{n, N}$, with fibers given by rescaling. Recall from Proposition 2.7 that $\overline{\mathcal{L}}_{n, N}$ is $n(N-2 n-1)$-dimensional. The set of periodic rescalings has $N$ parameters, so the dimension of $\mathcal{E}_{n, N}$ is $(n+1) N-n(2 n+1)$. Thus the number of independent constraints imposed on a periodic symmetric linear difference operator by specifying its monodromy to be - Id is the dimension of the symplectic group preserving $\mathcal{W}_{A}$, as one would predict from Lemma 6.7.

Proposition 6.8. Suppose that $A$ is in $\mathcal{E}_{n, N}(\mathbb{K})$. Fix arbitrarily an identification of the symplectic space $\left(\mathcal{K}(A), \mathcal{W}_{A}\right)$ with the standard symplectic space $\left(\mathbb{K}^{2 n}, \omega\right)$, and let $v_{i} \in \mathbb{K}^{2 n}$ be the image under this identification of the element $V^{i}(A)$ of $\mathcal{K}(A)$ defined in Corollary 6.3 . 
(i) The $v_{i}$ are $N$-antiperiodic.

(ii) $\left(\mathbb{K} v_{1}, \ldots, \mathbb{K} v_{N}\right)$ is an $(n, N)$-Lagrangian configuration.

(iii) There is a map $P: \mathcal{E}_{n, N}(\mathbb{K}) \rightarrow \overline{\mathcal{L}}_{n, N}(\mathbb{K})$, defined by

$$
P(A):=\text { the symplectic equivalence class of }\left(\mathbb{K} v_{1}, \ldots, \mathbb{K} v_{N}\right) .
$$

(iv) $P(A)$ and $P(-A)$ are opposite configurations.

Proof. The fact that the monodromy $M_{A}$ is -Id translates to the statement that $V^{i+N}(A)=-V^{i}(A)$, giving (i). For (ii), apply Lemmas 2.2 and 6.4(iii) and use the fact that $\mathcal{W}_{A}\left(V^{i}(A), V^{j}(A)\right)=\omega\left(v_{i}, v_{j}\right)$ by construction. For (iii), note that the symplectic equivalence class of $\left(\mathbb{K} v_{1}, \ldots, \mathbb{K} v_{N}\right)$ is independent of the choice of symplectic identification of $\mathcal{K}(A)$ with $\mathbb{K}^{2 n}$.

For (iv), use the facts that $\mathcal{K}(-A)=\mathcal{K}(A), V^{i}(-A)=-V^{i}(A)$, and $\mathcal{W}_{-A}=-\mathcal{W}_{A}$.

Theorem 3. $\quad$ (i) $P: \mathcal{E}_{n, N}(\mathbb{K}) \rightarrow \overline{\mathcal{L}}_{n, N}(\mathbb{K})$ is surjective.

(ii) $P(A)=P(\tilde{A})$ if and only if $\tilde{A}$ is a rescaling $\lambda^{-1} A \lambda^{-1}$ of $A$ by an $N$-periodic $\lambda$.

Proof. We proceed by a series of lemmas. For (i), fix an $(n, N)$-Lagrangian configuration $\left(\mathbb{K} x_{1}, \ldots, \mathbb{K} x_{N}\right)$. As usual, extend the representatives $N$-antiperiodically to $\left(x_{i}\right)_{i \in \mathbb{Z}}$ and write $\omega_{i j}$ for $\omega\left(x_{i}, x_{j}\right)$. In order to construct an operator $A$ in $\mathcal{E}_{n, N}(\mathbb{K})$ such that $P(A)$ is the class of $\left(\mathbb{K} x_{1}, \ldots, \mathbb{K} x_{N}\right)$, for all $i$ define

$$
a_{i}^{n}:=1 / \omega_{i-n, i} .
$$

Keeping in mind that $\left\{x_{i-n+1}, \ldots, x_{i+n}\right\}$ is a basis of $\mathbb{K}^{2 n}$, define $a_{i}^{n-1}, \ldots, a_{i}^{-n}$ by the equation

$$
a_{i}^{n} x_{i-n}+a_{i}^{n-1} x_{i-n+1}+\cdots+a_{i}^{-n+1} x_{i+n-1}+a_{i}^{-n} x_{i+n}=0 .
$$

Define $A$ by $(A V)_{i}:=a_{i}^{n} V_{n-i}+\cdots+a_{i}^{-n} V_{n+i}$. The next two lemmas concern this difference operator.

Lemma 6.9. $\quad$ (i) $A$ is non-degenerate, $N$-periodic, and symmetric.

(ii) For $1 \leqslant p \leqslant n$, the coefficients $a_{i}^{n-p}$ are given by

$$
a_{i}^{n-p}=\sum_{m=0}^{p-1} \sum_{0<p_{1}<\cdots<p_{m}<p}-(-1)^{m} \frac{\omega_{i-n, i+p_{1}} \omega_{i-n+p_{1}, i+p_{2}} \cdots \omega_{i-n+p_{m-1}, i+p_{m}} \omega_{i-n+p_{m}, i+p}}{\omega_{i-n, i} \omega_{i-n+p_{1}, i+p_{1}} \cdots \omega_{i-n+p_{m}, i+p_{m}} \omega_{i-n+p, i+p}},
$$

where the summand at $m=0$ is understood to be $-\omega_{i-n, i+p} / \omega_{i-n, i} \omega_{i-n+p, i+p}$.

Examples. Observe that (6.9) has $2^{p-1}$ summands. The first three cases are

$$
\begin{aligned}
& a_{i}^{n-1}= \frac{-1}{\omega_{i-n, i}}\left(\frac{\omega_{i-n, i+1}}{\omega_{i-n+1, i+1}}\right), \\
& a_{i}^{n-2}= \frac{-1}{\omega_{i-n, i}}\left(\frac{\omega_{i-n, i+2}}{\omega_{i-n+2, i+2}}-\frac{\omega_{i-n, i+1} \omega_{i-n+1, i+2}}{\omega_{i-n+1, i+1} \omega_{i-n+2, i+2}}\right), \\
& a_{i}^{n-3}=\frac{-1}{\omega_{i-n, i}}\left(\frac{\omega_{i-n, i+3}}{\omega_{i-n+3, i+3}}-\frac{\omega_{i-n, i+1} \omega_{i-n+1, i+3}}{\omega_{i-n+1, i+1} \omega_{i-n+3, i+3}}-\frac{\omega_{i-n, i+2} \omega_{i-n+2, i+3}}{\omega_{i-n+2, i+2} \omega_{i-n+3, i+3}}\right. \\
&\left.\quad+\frac{\omega_{i-n, i+1} \omega_{i-n+1, i+2} \omega_{i-n+2, i+3}}{\omega_{i-n+1, i+1} \omega_{i-n+2, i+2} \omega_{i-n+3, i+3}}\right) .
\end{aligned}
$$

Proof. Apply $\omega\left(x_{i-r}, \cdot\right)$ to (6.8) to obtain

$$
0=a_{i}^{n} \omega_{i-r, i-n}+a_{i}^{n-1} \omega_{i-r, i-n+1}+a_{i}^{n-2} \omega_{i-r, i-n+2}+\ldots+a_{i}^{-n} \omega_{i-r, i+n} .
$$

Consider the case $r=0$. By the Lagrangian condition, here only the leftmost and rightmost terms on the right hand side are non-zero. We obtain

$$
a_{i}^{-n}=1 / \omega_{i, i+n}=a_{i+n}^{n} .
$$

Thus $A$ is non-degenerate and satisfies the symmetry condition $a_{i}^{-\ell}=a_{i+\ell}^{\ell}$ for $\ell=n$. 
Now consider the cases $r= \pm p$ with $1 \leqslant p \leqslant n$. By the Lagrangian condition, for $r=-p$ only the leftmost $p+1$ terms on the right hand side are non-zero, while for $r=p$ only the rightmost $p+1$ terms are non-zero. We obtain

$$
\begin{aligned}
& a_{i}^{n-p}=\frac{-1}{\omega_{i-n+p, i+p}}\left(\frac{\omega_{i-n, i+p}}{\omega_{i-n, i}}+a_{i}^{n-1} \omega_{i-n+1, i+p}+\cdots+a_{i}^{n-p+1} \omega_{i-n+p-1, i+p}\right), \\
& a_{i}^{p-n}=\frac{-1}{\omega_{i-p, i+n-p}}\left(\frac{\omega_{i-p, i+n}}{\omega_{i, i+n}}+a_{i}^{1-n} \omega_{i-p, i+n-1}+\cdots+a_{i}^{p-1-n} \omega_{i-p, i+n-p+1}\right) .
\end{aligned}
$$

A straightforward induction argument from (6.12) gives (6.9): the first term of (6.12) is the $m=0$ term of (6.9), and the term $-a_{i}^{n-q} \omega_{i-n+q, i+p} / \omega_{i-n+p, i+p}$ of (6.12) gives those terms of (6.9) with $p_{m}=q$. A parallel argument from (6.13) yields a closed formula for $a_{i}^{p-n}$ :

$$
a_{i}^{p-n}=\sum_{m=0}^{p-1} \sum_{0<p_{1}<\cdots<p_{m}<p}-(-1)^{m} \frac{\omega_{i-p, i+n-p_{m}} \omega_{i-p_{m}, i+n-p_{m-1}} \cdots \omega_{i-p_{2}, i+n-p_{1}} \omega_{i-p_{1}, i+n}}{\omega_{i-p, i+n-p} \omega_{i-p_{m}, i+n-p_{m}} \cdots \omega_{i-p_{1}, i+n-p_{1}} \omega_{i, i+n}},
$$

where the summand at $m=0$ is understood to be $-\omega_{i-p, i+n} / \omega_{i-p, i+n-p} \omega_{i, i+n}$.

To finish proving that $A$ is symmetric, we must prove $a_{i}^{-\ell}=a_{i+\ell}^{\ell}$ for $0 \leqslant \ell<n$. Note that

$$
\left(p_{1}, p_{2}, \ldots, p_{m}\right) \mapsto\left(p-p_{m}, p-p_{m-1}, \ldots, p-p_{1}\right)
$$

is an involution of the index set of the inner summation in (6.9). Use this to verify that replacing $i$ by $i+n-p$ in (6.9) gives (6.14). This completes the proof of the lemma: the fact that $A$ is $N$-periodic is now immediate from $\omega_{i+N, j+N}=\omega_{i j}$.

Lemma 6.10. $\quad$ (i) $A$ lies in $\mathcal{E}_{n, N}(\mathbb{K})$.

(ii) The solutions $V^{i}(A)$ defined in Corollary 6.3 are given by $V_{j}^{i}(A)=\omega_{i j}$.

Proof. We begin with (ii). Abbreviate $V^{i}(A)$ by $V^{i}$. By (6.4), (6.7), and (6.11),

$$
\begin{aligned}
\left(V_{i-n}^{i}, V_{i-n+1}^{i}, \ldots, V_{i+n-1}^{i}, V_{i+n}^{i}\right) & :=\left(\omega_{i, i-n}, 0, \ldots, 0, \omega_{i, i+n}\right) \\
& :=\left(\omega_{i, i-n}, \omega_{i, i-n+1} \ldots, \omega_{i, i+n-1}, \omega_{i, i+n}\right) .
\end{aligned}
$$

Consider (6.10): since $i-r$ is arbitrary, we see that $\left(\omega_{i j}\right)_{j}$ lies in $\mathcal{K}(A)$. By the above identity, it has the same initial conditions as $V^{i}$, and so (ii) follows from Lemma 6.2

In light of Lemma 6.9(i), to prove (i) it suffices to prove that (6.8) has monodromy - Id. Because the $V^{i} \operatorname{span} \mathcal{K}(A)$, this reduces to $V_{j+N}^{i}=-V_{j}^{i}$ for all $i, j$. By (ii), this follows from $x_{j+N}=-x_{j}$.

At this point we have proven Theorem 3 (i): by Lemmas 6.4 and 6.10, the element $A$ of $\mathcal{E}_{n, N}(\mathbb{K})$ constructed in Lemma 6.9 has solutions $V^{i}(A)$ satisfying $\mathcal{W}_{A}\left(V^{i}, V^{j}\right)=V_{j}^{i}=\omega\left(x_{i}, x_{j}\right)$. Therefore by Lemma 2.1(iii) there is an element of $\operatorname{Sp}(2 n, \mathbb{K})$ carrying the $x_{i}$ to the $v_{i}$ of Proposition 6.8 , and so $P(A)$ is the class of the Lagrangian configuration originally given.

We now turn to Theorem 3 (ii). The fact that $P(A)=P(\tilde{A})$ if $A=\lambda \tilde{A} \lambda$ is immediate from Lemma 6.5 $\lambda$ is a symplectic map carrying $V^{i}(A)$ to a multiple of $V^{i}(\tilde{A})$. Conversely, suppose that $P(A)=P(\tilde{A})$. Reviewing Proposition 6.8, we find that this means there is a symplectic map $\Lambda$ from $(\mathcal{K}(A), \mathcal{W} A)$ to $\left(\mathcal{K}(\tilde{A}), \mathcal{W}_{\tilde{A}}\right)$ carrying $V^{i}(A)$ to a non-zero multiple of $V^{i}(\tilde{A})$, for all $i$. Define $\lambda$ by setting $\lambda_{i}^{-1}$ to be this multiple. Because the sequences $V^{i}(A)$ and $V^{i}(\tilde{A})$ are both $N$-antiperiodic, $\lambda$ is $N$-periodic.

Let $\hat{A}:=\lambda \tilde{A} \lambda$. By Lemma 6.5. $\lambda$ is a symplectic map from $\left(\mathcal{K}(\hat{A}), \mathcal{W}_{\hat{A}}\right)$ to $\left(\mathcal{K}(\tilde{A}), \mathcal{W}_{\tilde{A}}\right) \operatorname{carrying} V^{i}(\hat{A})$ to $\lambda_{i}^{-1} V^{i}(\tilde{A})$. Therefore $\lambda^{-1} \circ \Lambda$ is a symplectic map from $\left(\mathcal{K}(A), \mathcal{W}_{A}\right)$ to $\left(\mathcal{K}(\hat{A}), \mathcal{W}_{\hat{A}}\right)$ carrying $V^{i}(A)$ to $V^{i}(\hat{A})$. The following lemma shows that $A=\hat{A}$, completing the proof of Theorem 3 . 
Lemma 6.11. Let $A$ and $\hat{A}$ be elements of $\mathcal{E}_{n, N}(\mathbb{K})$. Write $a^{\ell}$ and $\hat{a}^{\ell}$ for the coefficients of $A$ and $\hat{A}, V^{i}$ and $\hat{V}^{i}$ for $V^{i}(A)$ and $V^{i}(\hat{A})$, and $\nu_{i j}$ and $\hat{\nu}_{i j}$ for the symplectic products $\mathcal{W}_{A}\left(V^{i}, V^{j}\right)$ and $\mathcal{W}_{\hat{A}}\left(\hat{V}^{i}, \hat{V}^{j}\right)$, respectively. The following statements are equivalent:

(i) $A=\hat{A}$, i.e., $a^{\ell}=\hat{a}^{\ell}$ for $0 \leqslant \ell \leqslant n$.

(ii) There exists a symplectic map $\sigma:\left(\mathcal{K}(A), \mathcal{W}_{A}\right) \rightarrow\left(\mathcal{K}(\hat{A}), \mathcal{W}_{\hat{A}}\right)$ such that $\sigma\left(V^{i}\right)=\hat{V}^{i}$ for all $i$.

(iii) $\nu_{i j}=\hat{\nu}_{i j}$ for all $i$ and $j$.

(iv) $V^{i}=\hat{V}^{i}$ for all $i$.

Proof. It is immediate that (i) implies (ii), (iii), and (iv), and (ii) implies (iii). By Lemma 2.1(iii), (iii) implies (ii), and (iii) and (iv) are equivalent by Lemma 6.4(ii). In order to prove that (iii) and (iv) imply (i), we will prove that for $0 \leqslant p \leqslant n$ the $a_{i}^{n-p}$ are given by (6.7) and (6.9) with $\nu_{i j}$ replacing $\omega_{i j}$.

For $p=0$, recall that by Lemma 6.4(iii), $a_{i}^{n}=1 / \nu_{i-n, i}$. For $p>0$ it suffices to prove that (6.12) holds with $\nu$ replacing $\omega$, and for this it suffices to prove that (6.10) holds with $\nu$ replacing $\omega$. This identity in turn results from applying $\mathcal{W}_{A}\left(V^{i-r}, \cdot\right)$ to (6.8) with $V^{\bullet}$ replacing $x_{\bullet}$, so finally we come down to proving the vector identity

$$
a_{i}^{n} V^{i-n}+a_{i}^{n-1} V^{i-n+1}+\cdots+a_{i+n-1}^{n-1} V^{i+n-1}+a_{i+n}^{n} V^{i+n}=0
$$

for all $i$. Because $V^{j}$ is itself in $\mathcal{K}(A)$, we know that the scalar identity

$$
a_{i}^{n} V_{i-n}^{j}+a_{i}^{n-1} V_{i-n+1}^{j}+\cdots+a_{i+n-1}^{n-1} V_{i+n-1}^{j}+a_{i+n}^{n} V_{i+n}^{j}=0
$$

holds for all $i$. To complete the proof, recall from Lemma 6.4(ii) that $V_{k}^{j}=-V_{j}^{k}$.

\section{THE CASE $N=2 n+3$}

In this section, let $\left(X_{1}, \ldots, X_{N}\right)$ be a generic complex $(n, N)$-Lagrangian configuration with representatives $\left(x_{1}, \ldots, x_{N}\right)$. Extend them to an $N$-antiperiodic sequence and write $\omega_{i j}$ for $\omega\left(x_{i}, x_{j}\right)$. We conclude the article with a discussion of the case $N=2 n+3$ : we generalize the five Gauss relations (1.3) on $\mathcal{L}_{1,5}(\mathbb{C})$ to $2 n+3$ relations on the $2 n+3$ basic symplectic cross-ratios of $\mathcal{L}_{n, 2 n+3}(\mathbb{C})$.

These relations are obtained by means of the symmetric linear difference operators associated to Lagrangian configurations in Theorem [3. The computations actually consist in solving the system of equations given by the condition that the operators have monodromy - Id. We remark that Theorem 2(i) may be obtained via the same method.

There are two sequences of non-trivial cross-ratios on $\mathcal{L}_{n, 2 n+3}$ : the $c_{i}$ of (1.4), and the $\gamma_{i, i+n+1}$ of (2.7), which we will abbreviate by $\gamma_{i}$. Both are $(2 n+3)$-periodic:

$$
c_{i}:=c_{i, i+1, i+n+1, i+n+2}=\frac{\omega_{i, i+n+1} \omega_{i+1, i+n+2}}{\omega_{i, i+n+2} \omega_{i+1, i+n+1}}, \quad \gamma_{i}:=c_{i-2, i, i+n, i+n+1}=\frac{\omega_{i-2, i+n} \omega_{i, i+n+1}}{\omega_{i-2, i+n+1} \omega_{i, i+n}} .
$$

We have $\gamma_{i}=c_{i-1} c_{i+n}$, so all cross-ratios on $\mathcal{L}_{n, 2 n+3}$ may be written in terms of the $c_{i}$. We will prove that over $\mathbb{C}$ these $2 n+3$ cross-ratios determine the equivalence class of their Lagrangian configuration (we expect that over $\mathbb{R}$ they determine it up to opposites). By Proposition $2.7 \mathcal{L}_{n, 2 n+3}(\mathbb{C})$ is $2 n$-dimensional, so the space of relations on the $c_{i}$ must have Krull dimension 3 . We conjecture that the $2 n+3$ relations we present generate the full space of relations.

If $\omega_{i, i+n}=1$ for all $i$, as in Section 3.3, then the representatives $x_{i}$ are said to be normalized. We begin with a general lemma permitting us to restrict our consideration to such representatives.

Lemma 7.1. For any complex $(n, N)$-Lagrangian configuration $\left(X_{1}, \ldots, X_{N}\right)$ with $N / \operatorname{GCD}(n, N)$ odd, there exist exactly $2^{\mathrm{GCD}(n, N)}$ normalized choices of representatives. 
Proof. Following Section 4.2 , let $\left(\tilde{x}_{1}, \ldots, \tilde{x}_{N}\right)$ be any representatives of $\left(X_{1}, \ldots, X_{N}\right)$, with corresponding symplectic products $\tilde{\omega}_{i j}$. Fix an $N$-periodic sequence $\chi_{i}$ such that $\chi_{i}^{2}=\tilde{\omega}_{i, i+n}$. Mimicking (4.9), set

$$
\lambda_{i}:=\frac{\chi_{i} \chi_{i+2 n} \chi_{i+4 n} \cdots \chi_{i+(q-1) n}}{\chi_{i+n} \chi_{i+3 n} \cdots \chi_{i+(q-2) n}}=\prod_{r=0}^{q-1} \chi_{i+r n}^{(-1)^{r}}
$$

where $q$ denotes $N / \operatorname{GCD}(n, N)$. Check that $\lambda_{i} \lambda_{i+n}=\tilde{\omega}_{i, i+n}$, so $x_{i}:=\tilde{x}_{i} / \lambda_{i}$ satisfies $\omega_{i, i+n}=1$.

The fact that $\lambda_{i}^{2}=\prod_{r=0}^{q-1} \tilde{\omega}_{i+r n, i+(r+1) n}^{(-1)^{r}}$ implies that each $q$-tuple $\left(\lambda_{i}, \lambda_{i+n}, \ldots, \lambda_{i+(q-1) n}\right)$ is determined up to a single choice of overall sign. The lemma follows.

Henceforth let $\left(X_{1}, \ldots, X_{2 n+3}\right)$ be a generic complex $(n, 2 n+3)$-Lagrangian configuration, and fix a normalized choice of representatives $x_{i}$. Recall from Section 3.3 the $(n+1)$-periodic sequence $a_{i}:=$ $\omega_{i, i+n+1}$ of symplectic diameters of normalized $(n, 2 n+2)$-configurations. The analog here is the $(2 n+3)$ periodic sequence of symplectic main diagonals, defined by the same formula as the $a_{i}$ :

$$
d_{i}:=\omega_{i, i+n+1} \text {. }
$$

Note that this notation is consistent with (1.3), and

$$
c_{i}=d_{i} d_{i+1} / d_{i-n-1}, \quad \gamma_{i}=c_{i-1} c_{i+n}=d_{i} d_{i+n} .
$$

Corollary 7.2. (i) If $3 \nmid n$, then $\left(X_{1}, \ldots, X_{2 n+3}\right)$ has two normalized choices of representatives: $\left(x_{i}\right)_{i}$ and $\left(-x_{i}\right)_{i}$. Both have the same $d_{i}$ : the configuration determines its main diagonals.

(ii) If $3 \mid n$, then $\left(X_{1}, \ldots, X_{2 n+3}\right)$ has eight normalized choices of representatives: $\epsilon_{i} x_{i}$, where $\epsilon_{i}= \pm 1$ and depends only on $i$ modulo 3. The corresponding main diagonals are $\epsilon_{i} \epsilon_{i+1} d_{i}$.

Proposition 7.3. Generic equivalence classes in $\mathcal{L}_{n, 2 n+3}(\mathbb{C})$ with the same cross-ratios $c_{i}$ are equal.

Proof. Let $x_{i}$ and $\tilde{x}_{i}$ be normalized representatives of two Lagrangian configurations having the same cross-ratios: $\tilde{c}_{i}=c_{i}$. It suffices to show that the $\tilde{x}_{i}$ may be chosen so that the two sets of main diagonals are the same, i.e., $\tilde{d}_{i}=d_{i}$, as then $\tilde{\omega}_{i j}=\omega_{i j}$ for all $i$ and $j$. Observe that

$$
c_{i-1} \gamma_{i+1}=d_{i-1} d_{i} d_{i+1} \text {, and for any } r, \frac{\gamma_{i} \gamma_{i+2 n} \cdots \gamma_{i+2 r n}}{\gamma_{i+n} \gamma_{i+3 n} \cdots \gamma_{i+(2 r-1) n}}=d_{i} d_{i+(2 r+1) n} .
$$

Write $n$ in the form $3 m+s$, where $s \in\{-1,0,1\}$. Take $r=m$ above and apply $(2 n+3)$-periodicity to see that $d_{i} d_{i+s}$ is determined by the cross-ratios. If $s= \pm 1$, dividing $c_{i-1} \gamma_{i+1}$ by this gives $d_{i-s}$ as a function of the cross-ratios. Thus for $3 \nmid m$, the cross-ratios determine the main diagonals.

If $3 \mid n$, i.e., $s=0$, taking $r=m$ gives $d_{i}^{2}$, and so the cross-ratios determine the main diagonals up to sign: $\tilde{d}_{i}=\delta_{i} d_{i}$ for some $\delta_{i}= \pm 1$. Using $\gamma_{i}=d_{i} d_{i+n}, \tilde{\gamma}_{i}=\gamma_{i}$, and $\operatorname{GCD}(n, 2 n+3)=3$, we find that $\delta_{i}$ depends only on $i$ modulo 3 . Applying $c_{i-1} \gamma_{i+1}=d_{i-1} d_{i} d_{i+1}$, we obtain $\delta_{i-1} \delta_{i} \delta_{i+1}=1$. Therefore by Corollary 7.2 it is possible to modify the $\tilde{x}_{i}$ so that $\tilde{d}_{i}=d_{i}$.

Recall now the difference operator $A$ constructed from the representatives $x_{i}$ in (6.8). In the normalized case the formula (6.9) for its coefficients simplifies, as the denominators are all 1:

$$
a_{i}^{n-p}=\sum_{m=0}^{p-1} \sum_{0<p_{1}<\cdots<p_{m}<p}-(-1)^{m} \omega_{i-n, i+p_{1}} \omega_{i-n+p_{1}, i+p_{2}} \cdots \omega_{i-n+p_{m-1}, i+p_{m}} \omega_{i-n+p_{m}, i+p},
$$

the summand at $m=0$ being $-\omega_{i-n, i+p}$. Observe that

$$
\omega_{i-n+p_{\ell-1}, i+p_{\ell}}= \begin{cases}d_{i-n+p_{\ell}-1} & \text { if } p_{\ell}-p_{\ell-1}=1, \\ d_{i+p_{\ell}} & \text { if } p_{\ell}-p_{\ell-1}=2, \\ 1 & \text { if } p_{\ell}-p_{\ell-1}=3, \\ 0 & \text { if } p_{\ell}-p_{\ell-1} \geqslant 4 .\end{cases}
$$


We write $a_{i}^{n-p}$ explicitly for $0 \leqslant p \leqslant 3$ :

$$
\begin{aligned}
& a_{i}^{n}=1, \quad a_{i}^{n-1}=-d_{i-n}, \quad a_{i}^{n-2}=d_{i-n} d_{i-n+1}-d_{i+2}, \\
& a_{i}^{n-3}=-d_{i-n} d_{i-n+1} d_{i-n+2}+d_{i-n} d_{i+3}+d_{i-n+2} d_{i+2}-1 .
\end{aligned}
$$

Proposition 7.4. The $2 n+3$ symplectic main diagonals $d_{i}$ of the normalized representatives $x_{i}$ of $\left(X_{1}, \ldots, X_{2 n+3}\right)$ satisfy the following $2 n+3$ polynomial relations (for $n=1$, take $a_{i}^{2}$ to be 0 ):

$$
0=a_{i}^{2}+d_{i+n+1} a_{i}^{1}+d_{i} a_{i}^{0}+a_{i+1}^{1} .
$$

Proof. Simply take $r=-(n+1)$ in (6.10).

Legendrian pentagons in $\mathbb{C P}^{1}$. This is $\mathcal{L}_{1,5}$, the Gaussian case discussed in Section 1.2. Here (7.1) reduces to (1.3). Using $d_{i}=c_{i-2} c_{i+1} / c_{i+2}$, the relations may be stated in terms of the $c_{i}$ :

$$
\frac{1}{c_{i}}+\frac{1}{c_{i-1} c_{i+1}}=1 \text {. }
$$

Legendrian heptagons in $\mathbb{C P}^{3}$. For $\mathcal{L}_{2,7}$, (7.1) reads

$$
d_{i-1} d_{i} d_{i+1}-d_{i-3} d_{i-1}-d_{i+1} d_{i+3}-d_{i}+1=0 .
$$

Using $d_{i}=c_{i-1} c_{i} c_{i+3} / c_{i-2} c_{i+1}$, this becomes

$$
\frac{1}{c_{i-3}}+\frac{1}{c_{i+3}}+\frac{1}{c_{i-2} c_{i+2}}-\frac{1}{c_{i-3} c_{i} c_{i+3}}=1 .
$$

Legendrian nonagons in $\mathbb{C P}^{5}$. For $\mathcal{L}_{3,9}$, (7.1) yields

$$
\begin{aligned}
d_{i-4} d_{i-3} d_{i+3} d_{i+4}-d_{i-4} d_{i-3} d_{i-1}-d_{i-3} d_{i} d_{i+3}-d_{i+1} d_{i+3} d_{i+4} & \\
-d_{i-4} d_{i+4}+d_{i-1} d_{i+1}+d_{i-3}+d_{i}+d_{i+3} & =0 .
\end{aligned}
$$

Using $c_{i}=d_{i} d_{i+1} / d_{i-4}, c_{i-1} c_{i+3}=\gamma_{i}=d_{i} d_{i+3}$, and $c_{i-1} \gamma_{i+1}=d_{i-1} d_{i} d_{i+1}$, this may be rewritten as

$$
\frac{1}{c_{i-1}}+\frac{1}{c_{i}}+\frac{1}{c_{i+1}}+\frac{1}{c_{i-1} c_{i+1}}+\frac{1}{c_{i-2} c_{i+2}}-\frac{1}{c_{i-4} c_{i} c_{i+1}}-\frac{1}{c_{i-2} c_{i} c_{i+2}}-\frac{1}{c_{i-1} c_{i} c_{i+4}}=1 .
$$

We close with a few general remarks. Note that for $3 \nmid n$, (7.1) can always be written as a rational relation on the $c_{i}$, because the $d_{i}$ are rational functions of the $c_{i}$. In light of the situation for $\mathcal{L}_{3,9}$, we expect that this is in fact true for all $n$. Also, although we have worked only over $\mathbb{C}$ in this section, it should be easy to show that the relations we have given on the $c_{i}$ hold also over $\mathbb{R}$.

Finally, let us reiterate our conjecture regarding (7.1). Because $\mathcal{L}_{n, 2 n+3}$ is $2 n$-dimensional and the cross-ratios $\left(c_{1}, \ldots, c_{2 n+3}\right)$ form a coordinate ring on it, the space of relations on the $c_{i}$ must be of Krull dimension 3 . We conjecture that the $2 n+3$ relations (7.1) generate the full relation space.

Acknowledgements. We are grateful to Sophie Morier-Genoud, Sergei Tabachnikov, and Richard Schwartz for enlightening discussions. C.H.C. was partially supported by Simons Collaboration Grants 207736 and 519533.

\section{REFERENCES}

[1] D. André, Terme général d'une série quelconque déterminée à la façon des séries récurrentes, Ann. Sci. École Norm. Sup. (2) 7 (1878), 375-408.

[2] V. I. Arnold, The Sturm theorems and symplectic geometry, Func. Anal. Appl. 19 (1985), 251-259.

[3] C. H. Conley, V. Ovsienko, Rotundus: triangulations, Chebyshev polynomials, and Pfaffians, Math. Intelligencer 40 (2018), no. 3, 45-50.

[4] H. S. M. Coxeter, Frieze patterns, Acta Arith. 18 (1971), 297-310.

[5] E. Falbel, I. Platis, The PU $(2,1)$ configuration space of four points in $S^{3}$ and the cross-ratio variety, Math. Ann. 340 (2008), no. 4, 935-962.

[6] R. Felipe, G. Mari-Beffa, The pentagram map on Grassmannians, to appear in Ann. Inst. Fourier, arXiv:1507.04765. 
[7] C. F. Gauss, Pentagramma Mirificum, Werke, Bd. III, 481-490; Bd. VIII, 106-111.

[8] M. Glick, P. Pylyavskyy, Y-meshes and generalized pentagram maps, Proc. London Math. Soc. 112 (2016), 753-797.

[9] B. Khesin, F. Soloviev, The geometry of dented pentagram maps, J. Europ. Math. Soc. 18 (2016), 147-179.

[10] I. Krichever, Commuting difference operators and the combinatorial Gale transform, Funct. Anal. Appl. 49 (2015), no. $3,175-188$.

[11] F. Labourie, What is... a cross-ratio? Notices AMS 55 (2008), 1234-1235.

[12] S. Morier-Genoud, Symplectic frieze patterns, arXiv:1803.06001.

[13] S. Morier-Genoud, V. Ovsienko, R. Schwartz, S. Tabachnikov, Linear difference equations, frieze patterns and combinatorial Gale transform, Forum Math., Sigma 2 (2014), e22 (45 pages).

[14] S. Morier-Genoud, V. Ovsienko, S. Tabachnikov, 2-Frieze patterns and the cluster structure of the space of polygons, Ann. Inst. Fourier 62 (2012), 937-987.

[15] A. Onishchik, R. Sulanke, Projective and Cayley-Klein geometries, Springer Monographs in Mathematics, SpringerVerlag, Berlin, 2006.

[16] V. Ovsienko, Self-dual differential operators and curves on Lagrangian Grassmannian that are subordinate to a loop, Math. Notes 47 (1990), no. 3-4, 270-275.

[17] V. Ovsienko, Partitions of unity in $\mathrm{SL}(2, \mathbb{Z})$, negative continued fractions, and dissections of polygons, Res. Math. Sci. 5 (2018), no. 2, Paper No. 21, 25 pp.

[18] V. Ovsienko, R. Schwartz, S. Tabachnikov, The pentagram map: A discrete integrable system, Comm. Math. Phys. 299 (2010), no. 2, 409-446.

[19] V. Ovsienko, R. Schwartz, S. Tabachnikov, Liouville-Arnold Integrability of the Pentagram Map on Closed Polygons, Duke Math. J. 162 (2013), 2149-2196.

[20] V. Ovsienko, S. Tabachnikov, Projective differential geometry old and new: From the Schwarzian derivative to the cohomology of diffeomorphism groups, Cambridge University Press, Cambridge, 2005.

[21] F. Soloviev, Integrability of the Pentagram Map, Duke Math. J. 162 (2013), 2815-2853.

[22] I. M. Yaglom, On linear subspaces of symplectic space, Trudy Sem. Vektor. Tenzor. Analizu 9 (1952), 309-318.

Charles H. Conley, Department of Mathematics, University of North Texas, Denton TX 76203, USA

E-mail address: conley@unt.edu

Valentin Ovsienko, CNRS, Laboratoire de Mathématiques U.F.R. Sciences Exactes et Naturelles Moulin de LA Housse - BP 103951687 REIMS CEDEX 2, France

E-mail address: valentin.ovsienko@univ-reims.fr 